This item was submitted to Loughborough's Research Repository by the author.

Items in Figshare are protected by copyright, with all rights reserved, unless otherwise indicated.

\title{
Modelling of mass transfer in gas-liquid stirred tanks agitated by Rushton turbine and CD-6 impeller: a scale-up study
}

PLEASE CITE THE PUBLISHED VERSION

\section{PUBLISHER}

Elsevier / ( ) The Institution of Chemical Engineers

VERSION

AM (Accepted Manuscript)

\section{LICENCE}

CC BY-NC-ND 4.0

\section{REPOSITORY RECORD}

Gimbun, Jolius, Chris D. Rielly, and Zoltan K. Nagy. 2009. "Modelling of Mass Transfer in Gas-liquid Stirred Tanks Agitated by Rushton Turbine and CD-6 Impeller: A Scale-up Study". figshare. https://hdl.handle.net/2134/4950. 
This item was submitted to Loughborough's Institutional Repository (https://dspace.lboro.ac.uk/) by the author and is made available under the following Creative Commons Licence conditions.

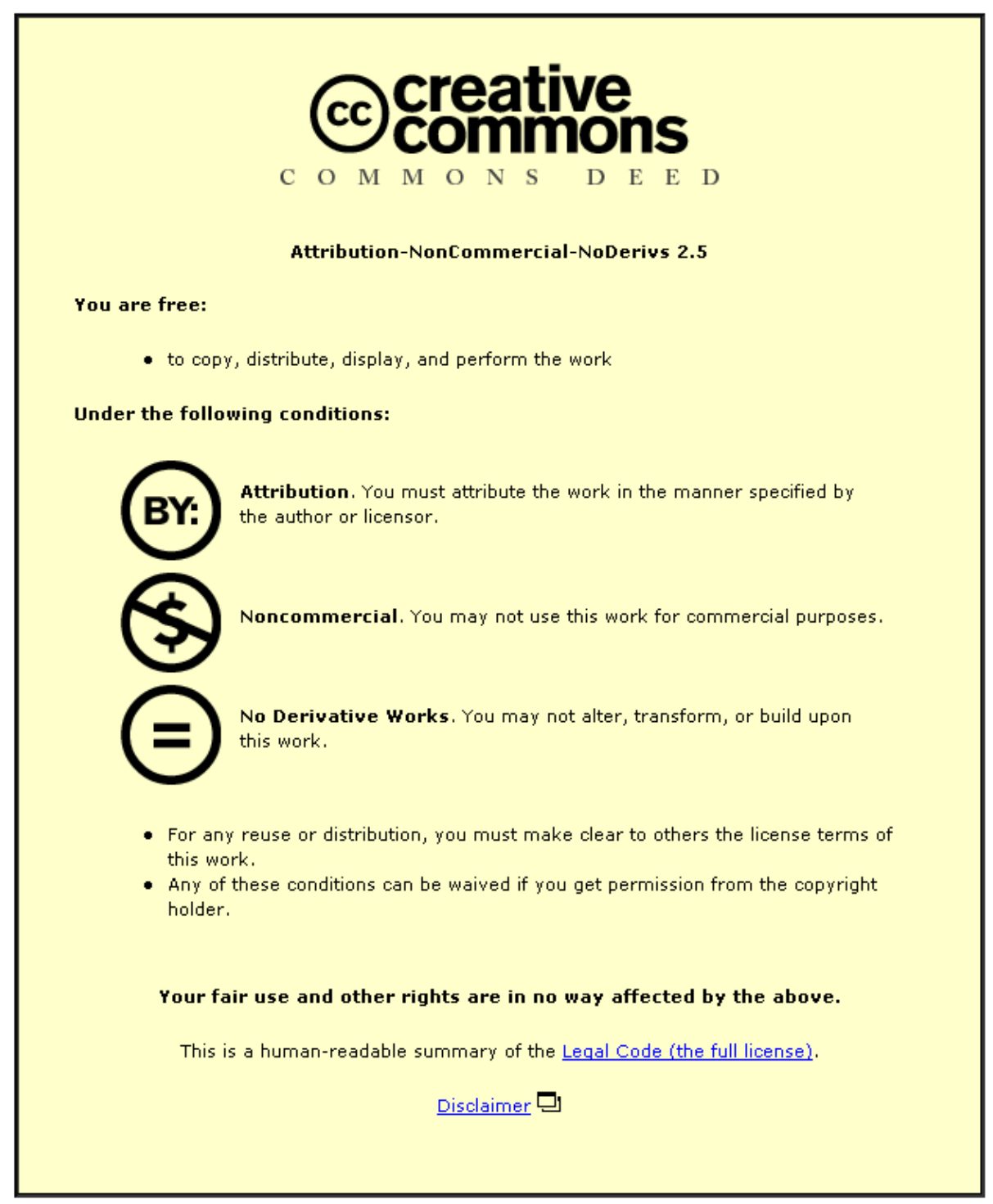

For the full text of this licence, please go to: http://creativecommons.org/licenses/by-nc-nd/2.5/ 


\title{
Modelling of Mass Transfer in Gas-Liquid Stirred Tanks Agitated by Rushton Turbine and CD-6 Impeller: A Scale-up Study
}

\author{
J. Gimbun, C. D. Rielly*, Z. K. Nagy \\ Dept. Chemical Engineering, Loughborough University, Leics, LE11 3TU, UK. \\ e-mail: c.d.rielly@lboro.ac.uk
}

\begin{abstract}
A combined computational fluid dynamics (CFD) and population balance model (PBM) approach has been applied to the simulation of gas-liquid stirred tanks agitated by (i) a Rushton turbine or (ii) a CD-6 impeller, operating at aeration numbers from 0.017 to 0.038 . The multiphase simulations were realised via an Eulerian-Eulerian two-fluid model and the drag coefficient of spherical and distorted bubbles was modelled using the Ishii-Zuber equations. The effect of the void fraction on the drag coefficient was modelled using the correlation by Behzadi et al. (2004). The local bubble size distribution was obtained by solving the PBM using the quadrature method of moments (QMOM). The local $k_{L} a$ was estimated using both the Higbie penetration theory and the surface renewal model. The predicted gas-liquid hydrodynamics, local bubble sizes and dissolved oxygen concentration were in good agreement with experimental measurements reported in the literature. A slight improvement in the prediction of the aerated power number was obtained using the non-uniform bubble size distribution resulting from the coupled CFD-PBM simulation. Evaluation of the prospective scale-up approaches indicates a higher probability of maintaining a similar level of mass transfer in a larger tanks by keeping the $P_{g} / V$ and $V V M$ constant. Considering its predictive capability, the method outlined in this work can provide a useful scale-up evaluation of gas-liquid stirred tanks.
\end{abstract}

Key words: gas-liquid; mass transfer; local bubble size; QMOM; CD-6; scale-up; CFD-PBM

\section{INTRODUCTION}

There are many industrial processes that involve gas-liquid dispersion in stirred tanks, e.g. in fine-chemicals manufacturing, or in biochemical fermentations. For economic and safety reasons, reliable models are needed for the scale-up and design of such reactors. One of the most important problems in modelling gas-liquid dispersions is the prediction of bubble size and gas-liquid interfacial area. As shown experimentally by many researchers (e.g. Montante et al., 2008; Barigou and Greaves, 1992; Laakkonen et al. 2005; 2007a) the distribution of bubble sizes varies inside the stirred tank depends on the spatial position. Generally, bubble 
sizes around the impeller discharge stream are the smallest due to breakage caused by high local energy dissipation rates. Furthermore, knowledge of bubble sizes is necessary in a twophase CFD model to calculate momentum exchange by drag. Hence, the population balance, phase continuity and momentum equations are coupled and should in principle be solved simultaneously. In addition, local bubble sizes and the local gas volume fraction are required for the calculation of the interfacial area, which is an important variable in designing an aerated stirred tank to achieve a required rate of gas-liquid mass transfer.

Many modelling studies on the gas-liquid stirred tanks have been performed in recent years, mostly using a uniform, mono-dispersed bubble size throughout the tank (e.g. Khopkar and Ranade, 2006; Sun et al., 2005; Wang et al., 2006; Morud and Hjertager, 1996; Deen et al., 2002; Scargiali et al., 2007). Generally, the CFD predictions of gas hold-up and mean flow are in fair agreement with experimental data, except around the impeller discharge. Previous studies have applied a variety of methods with uniform bubble sizes such as grid refinement, different drag laws and various turbulence models, but without complete success. Deen et al. (2001) evaluated the effects of different drag laws and grid refinement and found good predictions of the mean radial velocity but poor predictions of the gas axial velocity. Others such as Sun et al. (2005) and Wang et al. (2006) employed a $k-\varepsilon-A_{p}$ turbulence model without a complete success in predicting the two-phase flow. Scargiali et al. (2007) studied the influence of turbulent dispersion force, virtual mass, grid refinement and the prescribed bubble size on the holdup in a gas-liquid flow. They concluded that the grid size may significantly affect the prediction, but effects of the turbulent dispersion force and virtual mass were not very significant in determining the distribution of gas holdup. Khopkar and Ranade (2006) studied a gas-liquid stirred tank operating at different flow regimes and obtained a reasonable predictions of the gas hold-up and gassed power number, but only by employing the turbulent drag correlations by Brucato et al. (1998): their work showed over prediction of gas hold-ups around the lower and upper circulation loops.

Whereas it is possible to predict correctly the mean flow in a single phase stirred tank using any RANS based turbulence model, this performance has not yet been replicated for gas-liquid stirred tanks. The common practice of employing a uniform bubble size throughout the tanks is suspected to be the main reason for the poor prediction of the two-phase flow in stirred tanks. Of course, other factors such as the drag model for distorted and dense bubbles, turbulent drag laws, lift and other forces also cannot be ruled out. However, their effects appear to be secondary compared to that of an assumed uniform bubble size on the 
predominant momentum exchange mechanism of inter-phase drag coefficient, which directly affects the prediction of the local mean velocities and gas hold-up.

Early attempts to predict the local bubble size were performed using the population bubble density model (BDM) and a one-way coupled approach, e.g. as in the model of Bakker and Van den Akker (1994). In recent years, a coupled CFD-BDM has been employed to predict the local bubble size in gas-liquid stirred tanks by Lane et al. (2002, 2005), Kerdouss et al. (2006) and Moilanen et al. (2008). In most cases, the BDM is reported to give a satisfactory prediction of the local bubble size, but only by adjusting some of the empirical constants within the model. This practice is thought to be inappropriate because the model is unlikely to be fully predictive and hence cannot be applied to cases where the experimental data are not available. Lane et al. (2005), for example, introduced a correction factor of up to 3.5 for the turbulence dissipation rate, while Kerdouss et al. (2006) adjusted constants in the breakage and coalescence term in order to get good agreement with measurements reported by Alves et al. (2002). Lane et al. (2005) argue that the turbulent dissipation rate is not predicted well by the RANS $k-\varepsilon$ turbulence model. However, the correction factor that was applied is too large, considering the under prediction of turbulent dissipation rate by $k$ - $\varepsilon$ model is only around than 30\% (Ducoste and Clark, 1999). The formulation of the BDM itself is also questionable, since proper bubble breakage and coalescence kernels are not included. Instead all equations related to the bubble size are lumped together as a function of the critical Weber number and energy dissipation rate, without considering the probability and rate of bubblebubble and bubble-eddy collisions. As a consequence, the BDM is not thought to be a fully predictive model for simulation of gas-liquid dispersions in stirred vessels.

A full PBM has been employed to predict the local bubble size in stirred tanks, mostly using a discretisation based on the method of classes (MOC). Venneker et al. (2001) performed a one-way coupled PBM via MOC for a stirred tank bioreactor. Recently, a coupled CFD-PBM simulation using the MOC also has been performed by Montante et al. (2008), Moilanen et al. (2008) and Kerdouss et al. (2008). Moilanen et al. (2008) showed reasonable agreement for the predicted and measured local bubble size, based on fitted model constants in the breakage and coalescence terms from a previous multi-block study. Montante et al. (2008) presented a good prediction of the number mean bubble size without adjusting the constants of the kernels, however the Sauter mean diameter was consistently underpredicted by approximately 50\%. No comparison on the predicted bubble size were presented by Kerdouss et al. (2008). A fully predictive model should not require the tuning of model parameters for each case considered. One downside of the MOC is its computational 
demand since it requires more than 30 classes to get a good level of accuracy in the prediction of the evolution of the moments of the bubble size distribution.

The quadrature method of moments (QMOM) is based on solving equations for the moments of the bubble size distribution; the quadrature approximation overcomes the difficulties in obtaining a closed form solution for population balance equations involving breakage and coalescence. The QMOM requires considerably less computational effort than the MOC and also is capable of providing an accurate prediction with a relatively small number of quadrature points. Hence it is suitable for coupling with simulations of the twophase hydrodynamics. The QMOM has been applied previously to breakage and aggregation problems (e.g. Marchisio et al., 2003). Recently, Petitti et al. (2007) have employed the QMOM to solve the bubble dynamics for gas-liquid dispersion. In their work, bubble coalescence is not considered and only a simple breakage kernel is employed instead of one based on the physics of bubble breakup. No comparisons with experimental measurement were presented by Petitti et al. (2007). In the interest of a reduced computational effort, the QMOM method was selected to solve the population balance equation for bubble dynamics in aerated stirred tanks in this work.

The first part of this work focuses on the development of a modelling approach for gasliquid stirred tanks. For an initial comparison, the CFD simulation was performed assuming a constant bubble size throughout the tank. A coupled CFD-PBM was then performed to account for the spatially non-uniform bubble sizes inside the tank. The CFD prediction of the two-phase flow field was compared to experiments by Deen et al. (2002), whereas the results using the CFD-PBM approach were compared against measurements by Laakkonen et al. (2007a and b). After validation, the model was used to evaluate the local mass transfer coefficients inside the tank, and to study the reactor scale-up, especially from the mass transfer perspective, which is often vital in aerobic fermentations.

\section{MODELLING APPROACH}

\subsection{CFD modelling of two-phase flow}

The Eulerian-Eulerian approach is employed for gas-liquid stirred tanks simulation in this work, whereby the continuous and disperse phases are considered as interpenetrating media, identified by their local volume fractions. The volume fractions sum to unity and are governed by the following continuity equations: 
$\frac{\partial}{\partial t}\left(\alpha_{l} \rho_{l}\right)+\nabla \cdot\left(\alpha_{l} \rho_{l} \vec{u}_{l}\right)=0$

where $\alpha_{l}$ is the liquid volume fraction, $\rho_{l}$ is the density, and $\vec{u}_{l}$ is the velocity of the liquid phase. The mass transferred between phases is negligibly small and hence is not included in the right hand-side of eq.(1). A similar equation is solved for the volume fraction of the gas phase by replacing the subscript $l$ with $g$ for gas. The momentum balance for the liquid phase is:

$\frac{\partial}{\partial t}\left(\alpha_{l} \rho_{l} \vec{u}_{l}\right)+\nabla \cdot\left(\alpha_{l} \rho_{l} \vec{u}_{l} \vec{u}_{l}\right)=-\alpha_{l} \nabla P+\nabla \cdot \overline{\bar{\tau}}_{l}+\vec{F}_{\mathrm{lg}}+\alpha_{l} \rho_{l} \vec{g}+\vec{F}_{l i f t, l}+\vec{F}_{v m, l}$

where $\overline{\bar{\tau}}_{l}$ is the liquid phase stress-strain tensor, $\vec{F}_{l i f t, l}$ is a lift force, $\vec{g}$ is the acceleration due to gravity and $\vec{F}_{v m, l}$ is the virtual mass force. A similar equation is solved for the gas phase. $\vec{F}_{\mathrm{lg}}$ is the interaction force between phases, mainly due to drag. As pointed out by Scargiali et al. (2007) the effects of the turbulent dispersion, virtual mass and lift are almost negligible, despite a significant increase in computational expenses and convergence difficulties. Scargiali et al. (2007) found a minimal increase of the overall gas hold-up from $4.36 \%$ to $4.60 \%$ and from $4.36 \%$ to $4.67 \%$ by adding the effect of virtual mass and lift force respectively. They concluded that the effect of the drag force largely predominates in aerated stirred tanks. A similar conclusion was also drawn by many previous studies, e.g. Bakker and Van Den Akker, 1994; Morud and Hjertager, 1996; Lane et al., 2002; Kerdouss et al., 2006). It was therefore decided not to include the effect of the virtual mass and lift force in this work. Hence, $\vec{F}_{\mathrm{lg}}$ is represented by a simple interaction term for the drag force, given by:

$\vec{F}_{\mathrm{lg}}=-\frac{3 \rho_{l} \alpha_{g} \alpha_{l} C_{D}\left|\vec{u}_{g}-\vec{u}_{l}\right|\left(\vec{u}_{g}-\vec{u}_{l}\right)}{4 d_{b}}$

where $C_{D}$ is a drag coefficient and $d_{b}$ is the Sauter mean bubble diameter.

The drag model employed has a significant effect on the flow field of the aerated flow, as it is related directly to the bubble terminal rise velocity. Bubbles have a tendency to form a non-spherical shape, especially those with a diameter $>3 \mathrm{~mm}$. Therefore, the drag model of Ishii and Zuber (1979) was selected in this work, as it takes into account the drag of distorted bubbles:

$C_{D}=\max \left(\frac{24}{\operatorname{Re}_{b}}\left(1+0.15 \operatorname{Re}_{b}^{0.687}\right), \min \left(\frac{2}{3} \sqrt{E_{O}}, 8 / 3\right)\right)$ 
where the $\operatorname{Re}_{b}$ and $E_{O}$ are the bubble Reynolds number and Eotvos number, respectively. The drag for the ellipsoidal bubble regime is dependent on the bubble shape through the Eotvos number, which represents the ratio of gravitational to surface tension forces; for the spherical cap regime the drag coefficient is approximately $8 / 3$. The effect of the local bubble volume fraction on the drag coefficient is estimated using Behzadi et al.'s (2003) correlation as follows:

$C_{D, \text { dense }}=C_{D}\left(e^{3.64 \alpha}+\alpha^{0.864}\right)$

where the $C_{D}$ is the drag coefficient for isolated bubble estimated using eq.(4), whereas $C_{D \text {,dense }}$ is for the dense dispersion of bubbles. The drag model described above is not available as a standard option in FLUENT and hence it has been implemented via a userdefined subroutine.

The stirred tank grid was prepared with a headspace to accommodate liquid expansion due to aeration. The liquid surface was modelled as a freely expandable liquid surface and the top of the headspace region was set as a pressure outlet, rather than using a fictitious 'degassing boundary condition'. The mass balance between the gas outflow at the outlet boundary (above the headspace region) and the gas inflow at the sparger was satisfied. The PBM and mass transfer calculations did not include the headspace region.

It is also important to consider the formation of the bubble cavity behind the impeller blade. According to Lane et al. (2005), it is possible to model the gas cavity in the EulerianEulerian framework, providing a certain modification is made to the interphase exchange coefficient: the drag coefficient is set to turn into that for isolated bubble when the void fraction is higher than 0.7 , i.e. the cavity behind the blade behaves in a manner similar to an isolated bubble, rather than the dense bubble case. An attempt to use the dense drag bubble model for the cavity region has been tested, resulting in the disappearance of the bubble cavity behind the blade and an over-prediction of the gassed power number by more than $60 \%$. The mean radial velocity was also found to be over predicted. However, this issue has been successfully addressed by treating the cavity as an isolated bubble.

\subsection{Turbulence modelling}

The turbulence modelling uses the two-phase realizable $k$ - $\varepsilon$ model, in which both $k$ and $\varepsilon$ are allowed to have different values for each phase. The transport equations for the realizable two-phase $k-\varepsilon$ model are given in the Fluent manual (2006) and the standard values of the model parameters have been applied. The realizable $k-\varepsilon$ is considered to be a better model 
than the standard $k-\varepsilon$ for stirred tank flows (Gimbun, 2009), as it better accounts for flow features such as strong streamline curvature, vortices and rotation. The realizable $k-\varepsilon$ differs from the standard $k-\varepsilon$ model in two important ways: first it has a new formulation of turbulent viscosity and second it employs a new transport equation for the dissipation rate incorporating different model constants.

\subsection{Population balance modelling}

\subsubsection{QMOM formulation}

The QMOM is employed to solve the PBM and predict the evolution of the moments of the bubble size distribution. For breakage and coalescence only, the QMOM equation for the $k^{\text {th }}$ moment of a single well-mixed system is given by:

$$
\begin{aligned}
& \frac{\mathrm{d} \mu_{k}}{\mathrm{~d} t}=\underbrace{\sum_{i=1}^{N} w_{i} a\left(L_{i}\right) b\left(k, L_{i}\right)}_{\text {birth due to breakage }}+\underbrace{\frac{1}{2} \sum_{i=1}^{N} w_{i} \sum_{j=1}^{N} w_{j}\left(L_{i}^{3}+L_{j}^{3}\right)^{k / 3} \beta\left(L_{i}, L_{j}\right)}_{\text {birth due to coalescence }} \\
&-\underbrace{\sum_{i=1}^{N} w_{i} a\left(L_{i}\right) L_{i}^{k}}_{\text {death due to breakage }}-\underbrace{\sum_{i=1}^{N} w_{i} L_{i}^{k} \sum_{j=1}^{N} w_{j} \beta\left(L_{i}, L_{j}\right)}_{\text {death due to coalescence }}
\end{aligned}
$$

where $\beta\left(L_{i}, L_{j}\right), a\left(L_{i}\right)$ and $b\left(k, L_{i}\right)$ are the coalescence kernel, breakage kernel and daughter bubble distribution function, respectively. Full details of the QMOM can be found elsewhere e.g. McGraw (1997) and Marchisio et al. (2003). In this implementation, the solution of the weights $(w)$ and abscissas $(L)$ from the moments was obtained using the product difference algorithm of Gordon (1968). To reduce computation cost of these simulations a QMOM based on two quadrature points was applied.

There are many breakage and coalescence kernels available for bubbly flow, but they are essentially written in a similar form except some minor differences in the model constants or assumptions. The Prince and Blanch (1990) model has been proven to give a good prediction of bubble size in bubble columns (e.g. Shimizu et al., 2000; Podila et al., 2007). Some researchers (e.g. Chen et al., 2005; Bordel et al., 2006; Podila et al., 2007) made a comparison of the prediction of various kernels combinations including those proposed by Prince and Blanch (1990), Luo and Svendsen (1996), Luo (1993), Chesters (1991), MartinezBazan et al. (1999) and Lehr et al. (2002). Their findings suggest that there is no great difference between the mean flow, gas hold-up and bubble Sauter mean diameter $\left(d_{32}\right)$ predicted using different kernels although, there are some differences in the predicted bubble size distribution. The Luo and Svendsen (1996) breakup kernel was found to generate 
excessively small and large bubbles, due to its U-shaped daughter bubble distribution function (Podila et al., 2007). Podila et al. also pointed out the problem of the Luo (1993) coalescence kernel, which tends to yield large bubbles due to its high coalescence rates. Laakkonen et al. (2007a) and Moilanen et al. (2008) employed a modified version of Prince and Blanch's (1990) model for their work on gas-liquid stirred tanks, and they reported a good agreement with experimental measurement. Laakkonen et al. (2007a) has compared the prediction of two different kernels i.e. Lehr et al. (2002) and a modified version of Prince and Blanch models. Their findings suggest that Lehr model tends to under predict the local bubble size in gasliquid stirred tanks, even though it has been reported to produce an excellence prediction for bubble columns (Lehr et al., 2002). Based on these previous studies, the Prince and Blanch (1990) model has been employed to predict the bubble dynamics in this work.

\subsubsection{Modelling of bubble coalescence}

Bubble collisions may occur due to a variety of mechanisms, e.g. Prince and Blanch (1990) consider collisions arising from turbulence, buoyancy and laminar shear. In turbulent flow, bubble collisions are driven mainly by random motion of bubbles due to turbulent eddies. Bubbles of different sizes also have different rise velocities which may lead to collision. There is also a possibility for bubbles from a high liquid velocity region to collide with bubbles in slower section of the velocity field. The bubble collision frequency for a Newtonian fluid can be modelled following the approach proposed by Prince and Blanch (1990):

$$
\omega\left(L_{i}, L_{j}\right)=\underbrace{\pi\left(\frac{L_{i}}{2}+\frac{L_{j}}{2}\right)^{2}\left[u_{t}^{2}\left(L_{i}\right)+u_{t}^{2}\left(L_{j}\right)\right]^{0.5}}_{\text {turbulent }}+\underbrace{\pi\left(\frac{L_{i}}{2}+\frac{L_{j}}{2}\right)^{2}\left|u_{\infty}\left(L_{i}\right)-u_{\infty}\left(L_{j}\right)\right|}_{\text {bouyancy }}
$$

where $u_{t}(L)$ is the turbulent velocity in the inertial range of isotropic turbulence (Rotta, 1972) and $u_{\infty}\left(L_{i}\right)$ is the rise velocity of bubble given as a function of bubble size (Clift et al., 1978).

The bubble collision efficiency, $\lambda\left(L_{i}, L_{j}\right)$, is the probability of coalescence during a bubble-bubble collision between sizes $L_{i}$ and $L_{j}$. For Prince and Blanch's model, the bubble collision efficiency is given as a function of film drainage and bubble-bubble contact times:

$$
\lambda\left(L_{i}, L_{j}\right)=\exp \left(\frac{-\ln \left(h_{o} / h_{f}\right) \sqrt{\left(L_{i j} / 2\right)^{3} \rho_{l} / 16 \sigma}}{\left(L_{i j} / 2\right)^{2 / 3} / \varepsilon^{1 / 3}}\right)
$$


where $L_{i j}=2\left(1 / L_{i}+1 / L_{j}\right)^{-1}, h_{o}$ is the initial film thickness and $h_{f}$ is the final thickness at which the film rupture occur. The value of $10^{-4} \mathrm{~m}$ for $h_{o}$ and a value of $10^{-8} \mathrm{~m}$ for $h_{f}$ from Prince and Blanch (1990) was used throughout this work. The bubble coalescence kernel, $\beta\left(L_{i}, L_{j}\right)$, is a product of bubble collision efficiency, from eq.(7), and collision frequency from eq.(8).

$\beta\left(L_{i}, L_{j}\right)=\lambda\left(L_{i}, L_{j}\right) \omega\left(L_{i}, L_{j}\right)$

\subsubsection{Modelling of bubble breakage}

Prince and Blanch (1990) considered the bubble break-up to be caused by collisions with turbulent eddies of sizes equal to, or smaller than, the bubble size. They argued that eddies smaller than 0.2 times the bubble diameter are unlikely to contribute significantly to the overall break-up rate and set the lower limit of the effective turbulent eddies as $0.2 L$. They considered only eddies having a velocity larger than the critical velocity, $u_{c i}$, where the disruptive force due to the kinetic energy of the eddy and the cohesive force due to surface tension balance each other. The break-up rate is given as a product of the collision rate of bubbles with turbulent eddies, $\theta_{i e}$, and the break-up efficiency, $\kappa_{i}$. According to Prince and Blanch (1990), the bubble break-up rate is given by the expression:

$a\left(L_{i}\right)=\theta_{i e} \kappa_{i}$

The collision rate of bubbles with turbulent eddies is given by Kennard (1938):

$\theta_{i e}=n_{i} n_{e} S_{i e}\left(u_{t i}^{2}+u_{t e}^{2}\right)^{0.5}$

where $n_{i}, n_{e}$ and $S_{i e}$ are the number of bubbles per unit volume, number of eddies per unit volume and collision cross-sectional area, respectively. The $u_{t i}$ is the turbulent velocity in the inertial range of isotropic turbulence (Rotta, 1972) and the eddy velocity, $u_{t e}$, of a size $L_{e}$ is also calculated analogously to Rotta (1972). The eddy size may be expressed using Kolmogorov's (1941) theory of isotropic turbulence as $L_{e}=\left(v_{l}^{3} / \varepsilon\right)^{1 / 4}$.

The break-up efficiency, $\kappa_{i}$, is given by (Kennard, 1938; Prince and Blanch, 1990):

$\kappa_{i}=\exp \left(u_{c i}^{2} / u_{t e}^{2}\right)$

where the $u_{c i}$ is the critical eddy velocity necessary to break a bubble of diameter $L_{i}$, given by Shimizu et al. (2000).

Prince and Blanch's (1990) break-up model does not include the daughter bubble size distribution. The daughter bubble distribution function, $b(L, \lambda)$, determines the number and 
size of the daughter particle, $L$, after the breakage event of particle size $\lambda$. Here, a uniform breakage function was selected with binary breakage to form similar particle sizes.

$b(L \mid \lambda)=\frac{6 L^{2}}{\lambda^{3}}$

There is a high possibility of non-binary breakage for liquid-liquid systems where the internal viscosity of the dispersed phase can lead to multiple daughter drops (Andersson and Andersson, 2006). However the assumption of binary break-up is considered valid for bubbles, since the air viscosity is low. Furthermore, a recent study by Andersson and Andersson (2006) revealed that more than 95\% of bubble break-ups involved binary breakage.

The population balance model was solved using user-defined scalars to represent the moments, weights and abscissas and was implemented via user-defined subroutine written in $\mathrm{C}$ language. All the breakage and coalescence kernel were implemented without adjusting any of the model constants. The user-defined subroutine was compiled within the commercial CFD code, FLUENT 6.3 and was available as an add-on program after the compilation; hence a fully coupled CFD-PBM simulation could be performed.

\subsection{Modelling of $k_{L} a$ and oxygen transfer rate}

Many empirical scale-up rules and correlations have been developed to calculate the volumetric mass transfer coefficient, $k_{L} a$, in aerated stirred tanks. However, the existing correlations are only capable of calculating the average $k_{L} a$ value in the tank and not the local values. Information about the local $k_{L} a$ is important in the study of gas-liquid stirred tanks to spot the occurrence of 'dead zones', where very little mass transfer occurs. Ideally, achieving a uniform $k_{L} a$ and uniform driving force is desirable during scale-up of aerated stirred tanks. Whilst this maybe the case for laboratory scale stirred tanks, it is not always true for larger scale tanks, which can suffer from zones of oxygen depletion, particularly where there is an oxygen sink, e.g. through chemical reaction.

Assuming a spherical bubble, the local interfacial area per unit volume may be calculated from

$a=\sum_{i} \pi d_{b, i}^{2} n_{i}$

where $d_{b, i}$ is the bubble size and $n_{i}$ is number of bubbles of size $d_{b, i}$ per unit volume of dispersion. The bubble sizes and numbers of bubble used in the calculation of the interfacial area were obtained from the CFD-PBM simulation, directly from the weights and abscissas 
used in the QMOM. Bubbles with diameters greater than around $3 \mathrm{~mm}$ (for air-water) are ellipsoidal, with an aspect ratio which may be calculated as a function of the Eotvos number from the correlation of Wellek et al. (1966)

$$
R=1+0.163 E_{O}^{0.757}
$$

which was developed originally for liquid-liquid dispersions. Guet et al. (2005) compared eq.(15) with their experimental measurements obtained using a four-point optical fibre probe and reported that Wellek et al.'s (1966) correlation is applicable for bubbles. In this work the interfacial area for small bubble $\left(d_{b} \leq 1 \mathrm{~mm}\right)$ was estimated from eq.(14), whilst the bigger bubbles $\left(d_{b}>1 \mathrm{~mm}\right)$ were assumed to be as oblate spheroids and their surface area was calculated using $R$ from eq.(15). Even larger bubbles $\left(d_{b}>5 \mathrm{~mm}\right)$ may not form perfect oblate ellipsoids in turbulent flow, however, eq.(15) is a step towards improved bubble shape prediction.

Penetration theory (Higbie, 1935) and the surface renewal model (Danckwerts, 1951) are two common methods of calculating $k_{L}$ when the bubble size is known. Higbie's (1935) penetration theory results in an average mass transfer coefficient for each bubble size given by:

$$
k_{L, i}=\frac{2}{\sqrt{\pi}} \sqrt{\frac{D_{l} u_{\text {slip }}}{d_{b, i}}}
$$

where $u_{\text {slip }}$ and $D_{l}$ are the bubble slip velocity and diffusion coefficient, respectively. The slip velocity can be obtained from the difference in phase velocities from an Eulerian-Eulerian two-fluid CFD simulation. Thus local values of $k_{L} a$ were calculated from:

$$
k_{L} a=2\left(\pi D_{l}\right)^{0.5} \sum_{i} d_{b, i}^{1.5} u_{s l i p}^{0.5} n_{i}
$$

Danckwerts (1951) suggested a refinement of the penetration model by assuming that $k_{L}$ is related to the average surface renewal rate resulting from exposure of the bubble interface to turbulent eddies with a variable contact time. Danckwerts suggested the surface renewal model as follows:

$$
k_{L}=\sqrt{D_{l} s}
$$

where $s$ is the fractional rate of surface-element replacement. Lamont and Scott (1970) assumed that the small scale turbulent motion, which extends from smallest viscous motion to inertial ones, affects the rate of mass transfer. Consequently, $s$ can be calculated using Kolmogorov's theory of isotropic turbulence. They suggested the eddy cell model as follows: 


$$
k_{L}=K D_{l}^{0.5}\left(\frac{\varepsilon_{l}}{v_{l}}\right)^{0.25}
$$

where $\varepsilon_{l}$ is the turbulence dissipation rate in the liquid phase, $v_{l}$ is the liquid dynamic viscosity and $K=0.4$ is the model constant. Combining $k_{L}$ and $a$ gives another equation for calculating the volumetric mass transfer coefficient:

$$
k_{L} a=K \pi D_{l}^{0.5}\left(\frac{\varepsilon_{l}}{v_{l}}\right)^{0.25} \sum_{i} d_{b, i}^{2} n_{i}
$$

The local oxygen transfer rate can be estimated from the following relations once the local $k_{L} a$ has been determined,

$N_{o}=k_{L} a\left(C_{o}^{*}-C_{o}\right)-r_{o}$

where $C_{o}^{*}$ is the oxygen solubility in the liquid phase, $C_{o}$ is the oxygen concentration in the liquid phase and $r_{o}$ is the specific oxygen consumption rate. The transport equation for dissolved oxygen mass fraction was also solved as a user-defined scalar implemented as a user-defined subroutine, with the sink terms given in the right had side of eq.(21) above.

\subsection{Tank geometry and numerical strategy}

Two scales, 14L and 200L, of aerated stirred tanks containing a Rushton turbine, studied by Laakkonen et al. (2007a) were considered for the CFD-PBM modelling. Gas was injected through a sparger ring at a flow rate ranging from 0.29 to $0.7 \mathrm{vvm}$ which is treated as a continuous source of gas (velocity inlet) in the CFD simulation. First, a two-phase CFD simulation was performed assuming a uniform bubble diameter throughout the tank. The interphase drag coefficient was estimated using the standard Schiller-Naumann drag model. The CFD simulation was performed using a half-tank domain consisting of about $225 \mathrm{k}$ hexahedral cells. A finer mesh was employed around the impeller up to 15 nodes placed along the impeller blade height. According to Derksen et al. (1999), a grid with eight or less nodes along the impeller blade height may not be able to resolve the vortex core structure correctly and hence can give errors in the predicted mean flow field. The impeller movement was modelled using a multiple reference frame and the Eulerian-Eulerian approach was employed for the multiphase modelling. The turbulence was modelled using the two-phase realizable $k$ - $\varepsilon$ model described in a previous section. Transient solvers with a second-order spatial interpolation scheme were also applied for the final simulation in order to minimise the amount of numerical diffusion. The iteration residual was set to fall below $1 \times 10^{-4}$ at each time 
step to achieve good convergence. The volume average of the gas void fraction at the rotating zone (impeller region) was also monitored and the iterations were only halted once a constant value was observed. A grid sensitivity study was performed prior to the final grid selection using three different meshes: coarse ( $165 \mathrm{k}$ with 6 nodes at impeller blade height), intermediate (225k with 11 nodes at impeller blade height) and fine (335k with 13 nodes at impeller blade height). It was found that a domain consisting of $225 \mathrm{k}$ cells yielded a grid independent solution (see Fig. 1).

\section{RESULTS AND DISCUSSION}

\subsection{Prediction of Gas-Liquid Hydrodynamics}

First, the CFD simulations were validated against experimental data using the two-phase PIV measurements reported by Deen et al. (2002) for a stirred tank with $F l_{g}=Q_{g} / N D^{3}=0.0296$. The impeller speed for Laakkonen's geometry was set to $513 \mathrm{rpm}$ to ensure the aeration number stayed at $F l_{g}=0.0296$ so that a sensible comparison between the CFD prediction and the experimental measurement from Deen et al. (2002) could be made.

The simulation was performed initially by assuming a constant bubble size of $3.5 \mathrm{~mm}$ throughout the tank. The bubbles were assumed to be spherical and the Schiller and Naumann (1935) drag model was employed to estimate the drag coefficient. The CFD results were timeaveraged over all blade angles and compared with Deen et al.'s (2002) PIV measurements. For easier comparison, the results for the mean velocities were normalised using the impeller tip velocity $\left(V_{\text {tip }}\right)$. Despite the assumption of a constant bubble size and spherical bubbles, the predictions (marked as CFD constant) shown in Figs. 2 and 3 are reasonably close to the experimental data. The differences can be explained by the neglect of bubble coalescence and break-up caused by the turbulent flow induced by the rotating impeller. These mechanisms are not considered in the case where a uniform bubble size is assumed throughout the tank.

A simulation using a non-uniform bubble size was next performed to evaluate these effects on the CFD predictions. The local bubble sizes were estimated using the population balance model, which tracks the moments of the bubble size distribution. The local Sauter mean diameters, obtained from the ratio of the third and second moments, were then passed into the CFD simulation and used for the two-phase flow modelling. The CFD-PBM simulations were performed using two different drag models: (i) the hard sphere drag model of Schiller and Naumann (1935) (a default FLUENT model) and (ii) another that takes into account the drag of distorted bubbles (Ishii and Zuber, 1979) and dense bubble effect (Behzadi et al., 2003). As expected, results obtained from the CFD-PBM modelling were slightly better compared to 
those obtained using a constant bubble size. The prediction of axial gas velocities below the impeller $(2 z / W<1)$ and the peak liquid radial velocities were in fair agreement with Deen $e t$ al.'s (2002) data. Using the non-spherical drag model (CFD-PBM-IZ) in the CFD-PBM approach further improved the results. This is due to the fact that the effect of local bubble sizes on the two-phase flow is mainly via the inter-phase exchange coefficient, which depends on the drag model. The Schiller-Naumann model is suitable for spherical rigid bubbles, but in comparison, the Ishii-Zuber model predicts drag coefficients for the spherical, ellipse and cap bubble regime. The difference observed between the flow fields predicted using a spherical drag model and the one that accounts for distorted bubbles is small for the cases considered in this paper, due to the proximity of the analysed region to the impeller tip. In this region, the bubble size is mainly below $3 \mathrm{~mm}$ and hence bubbles can be assumed to be approximately spherical. However, because of the better prediction of the gas and liquid mean velocities, the CFD-PBM-IZ was selected and used for the remainder of this work. The remaining discrepancy in the result predicted by CFD-PBM-IZ method might be due to minor differences in the tank geometry used by Deen et al. (2002) and Laakkonen et al. (2007a) (the geometry used for the CFD work reported here). For instance Deen et al. (2002) used a dished bottom tank and had a slightly different impeller geometry $\left(W=L_{D}=0.25 D\right)$ whereas Laakkonen's work used a flat bottomed tank and a standard Rushton turbine. The inherent limitation of the Eulerian-Eulerian model which can only use a single bubble size $\left(d_{32}\right)$ at any spatial location at any given time is also thought to affect the accuracy of CFD prediction. A more accurate modelling approach of the gas-liquid flow would employ the real bubble size distribution at each spatial position inside the tank, however such model would require a higher implementation complexity and would be computationally more intensive to run. Therefore, a combined CFD-PBM model employing only the $d_{32}$ is thought to be a more efficient solution for a gas-liquid flow at present with the aim of employing the developed approach as a practical design tool.

\subsection{Prediction of the Aerated Power Number}

Prediction of the gassed power input by integrating the dissipation rate over the tank volume is known to provide an underestimate of the power input (in the cases shown here, by between 35-44\%). Therefore the $P_{g}$ in this work was calculated from the moment acting on the shaft and impeller or baffles and tank wall. The calculated torque, $\Gamma$, is then related to the power input by, 
For a Rushton turbine Bujalski et al. (1987) suggested the following correlation for estimation of the ungassed power number:

$N_{p 0}=\frac{P_{0}}{\rho_{l} N^{3} D^{5}}=2.512\left(\frac{\Delta t}{D}\right)^{-0.195} T^{0.063}$

where $\Delta t$ is the impeller thickness and $T$ is the tank diameter (m). Smith (2006) proposed the following correlation for the relative power draw, $P_{g} / P_{0}$, for stirred tanks agitated by a Rushton turbine, based on the measurements of Warmoeskerken and Smith (1982) and Gezork et al. (2000):

$P_{g} / P_{0}=0.18 \mathrm{Fr}^{-0.2} \mathrm{Fl}_{g}^{-0.25}$,

where $\mathrm{Fr}$ and $\mathrm{Fl}_{g}$ are the Froude number and the aeration number, respectively. Myers et al. (1999) performed extensive experiments in single phase and aerated stirred tank with a CD-6 impeller; they reported that, on gassing, the $P_{g} / P_{0}$ of a Rushton turbine drops significantly compared to that of a CD-6 impeller. In this study the CFD predictions were compared with measured $P_{g} / P_{0}$ obtained from Myers et al. (1999) for the CD-6 impeller, and using eq.(24) for the Rushton turbine, together with eq.(23).

The $P_{g} / P_{0}$ ratio is shown to be predicted reasonably well using the assumption of a constant bubble sizes throughout the tank (see Tables 1 and 2). There is a small improvement in the prediction of $P_{g} / P_{0}$ when a non-uniform bubble size is employed using the CFD-PBM method, especially for cases $1,4,5$ and 6 for which the uniform bubble sizes used for the initial simulation differed significantly from those calculated using the PBM. The bubble sizes for cases 2 and 3 were known from Laakkonen et al. (2007a), and mean values were used for these initial CFD simulations. Consequently, the CFD predictions using uniform bubble sizes for cases 2 and 3 are much closer to the values estimated from eq.(24). The results suggest that the $P_{g} / P_{0}$ can be predicted reasonably well using the uniform bubble size assumption with bubble size close to the experimental mean values. However, the CFD-PBM method is a more suitable approach for predicting the relative power number in cases when the mean bubble size is not known beforehand.

\subsection{Prediction of Local Bubble Size and Mass Transfer Coefficient}

CFD-PBM simulations were performed using a user-defined subroutine compiled within FLUENT. The Prince and Blanch (1990) breakage and coalescence kernels were employed to predict the bubble dynamics throughout the tank, using literature values of the model 
constants. The volume-average Sauter mean diameter, $d_{32}$, in the impeller region was used as a convergence indicator in these simulations.

Figures 4 and 5 show that the local bubble sizes predicted by the CFD-PBM simulation for both the smaller and the larger tanks are in good agreement with the experiments by Laakkonen et al. (2007a). The smallest bubbles can be observed around the impeller, where the dissipation rates are a maximum, whereas the largest bubbles are found below the impeller, just above the sparger, due to the combination of a high void fraction and low dissipation rates. Some discrepancies in the local bubble size predictions can be observed, possibly due to the well-known under-prediction of the energy dissipation rates by the $k-\varepsilon$ model - the evolution of the bubble size depends mainly on the dissipation rates and the gas void fraction. The CFD-PBM approach is also capable of responding to changes in operating conditions. For instance, case 1, which considers a lower impeller speed, produces larger bubbles compared to case 2, where the impeller speed is higher (see Table 1).

Using the local bubble size obtained, the local $k_{L} a$ can be estimated using Higbie's penetration theory, or the surface renewal model of Danckwerts. The latter gave a significantly higher value of $k_{L} a$ around the impeller region (see Fig. 6) due to its sensitivity towards high dissipation rates. Higbie's method return a higher local $k_{L} a$ in the bulk region, where the dissipation rates were very low; the two methods show slightly different sensitivities to the local dissipation rate and bubble size. The maximum local $k_{L} a$ values for the larger tank were significantly smaller (roughly 50\% less) than for the smaller tank due to the larger mean bubble size, which consequently reduced the interfacial area. The local $k_{L} a$ contour map also revealed a large dead zone in the bottom region of the tank due to the poor gas dispersion produced by the Rushton turbine. This can be addressed by employing a better gas dispersion impeller such as the CD-6, as shown in Fig. 7A. The CD-6 impeller is a concave type impeller which is available commercially from Chemineer and has been studied extensively by many researchers (e.g. Myers et al., 1999). There are several reason why the CD-6 disperses bubbles much better than the Rushton turbine. Firstly, the CD-6 pumps the fluid slightly downward around the impeller discharge region, whereas the Rushton turbine pumps slightly upward (see Fig. 7A), which then contributes to poor circulation of bubbles in the lower region. Secondly, the concave shape of the CD-6 is designed to produce a smaller gas cavity behind the impeller blade (see Fig. 7B) leading to less reduction in the aerated power number in comparison to the Rushton turbine. 
By analogy with experimental measurements, which often assume a well-mixed liquid phase, a global mean $\left\langle k_{L} a\right\rangle$ was estimated by monitoring the volume-averaged oxygen concentration, $C_{o}(t)$, throughout the simulation, from

$$
\ln \left(\frac{C_{o}^{*}-C_{o}(t)}{C_{o}^{*}-C_{o}(0)}\right)=-\left\langle k_{L} a\right\rangle t
$$

where $C_{o}^{*}$ is the oxygen solubility in water. In the cases discussed here, estimated mixing times from the correlation by Grenville and Nienow (2004), were about one order of magnitude greater than $\left\langle k_{L} a\right\rangle^{-1}$, indicating the liquid phase was well-mixed. Values of $\left\langle k_{L} a\right\rangle$ were obtained from the slopes of the graphs obtained by plotting the left hand side of eq.(25) against time. Fig. 8 shows the evolution of the dissolved oxygen concentration, [DO], calculated using the Higbie and Danckwerts methods, respectively. The predicted $C_{o}(t)$ profile is in good agreement with the experimental measurement from Laakkonen et al. (2007b) especially when an oblate spheroid shape is considered for the larger bubbles. As expected, the discrepancy is much bigger when bubbles assumed to be in spherical shape throughout the tank which may not be correct for diameters $>3 \mathrm{~mm}$. Furthermore, the Eulerian-Eulerian simulation works with a single slip velocity, despite the existence of a range of bubble sizes; this may introduce some discrepancy in the local $k_{L} a$ and the [DO] evolution. However, this simplification is necessary in order to keep the computational demand minimal. Moreover, the two-phase model can get excessively complicated and expensive to compute when individual bubble sizes with separate slip velocities are considered. Due to its better prediction of the [DO] evolution, the combined spherical and oblate spheroid model is applied for the remainder of this work.

Higbie's method is consistently found to have a slightly faster oxygen transfer rate than the Danckwerts's method for a smaller vessel (see Fig. 9A), where the mean bubble size is less than $3 \mathrm{~mm}$, but the difference becomes almost insignificant for the larger vessels (see Fig. 9B) when the mean bubble size is about $4 \mathrm{~mm}$. This is reflected in the calculated values of the mean $\left\langle k_{L} a\right\rangle$ shown in Tables 3, for cases 2 and 4. Furthermore, Danckwerts' model tend to have a faster oxygen transfer rate than the Higbie model when the mean bubble size is larger than $5 \mathrm{~mm}$ (see Fig. 8). This phenomenon can be explained by the sensitivity of the Higbie's model to small bubble sizes which are formed in great numbers for the smaller vessel. 
The [DO] evolution was recorded at three different locations inside the tank namely the dead zone below the sparger, the impeller region and bulk region above the impeller. Only a small amount of variation was found between the $\left\langle k_{L} a\right\rangle$ value estimated using the [DO] evolution recorded at these three different locations (see Fig. 10), hence the remaining discussion focuses only on the data recorded at impeller discharge, where the majority of experimental measurements have been obtained. In eq. (25), and often in experimental measurements of the mass transfer coefficient, it is assumed that the dissolved oxygen concentration, $[\mathrm{DO}]$ is uniform. Results from the CFD-PBM simulation suggests that this assumption is applicable for these lab and pilot scale gas-liquid vessels, without the presence of oxygen sink (i.e. reaction or micro-organism respiration). It is important to note that, the assumption of uniform [DO] may not be valid in a gas-liquid bioreactor even at small scale, depending on the local rate of consumption of dissolved oxygen. In such a case, the [DO] may fall towards zero in some dead regions leading to a severe mass transfer limitation. The wellmixed assumption is also less likely to be correct with increasing scale of operations (Schuetze and Hengstler, 2006), especially when dealing with industrially sized vessels. Thus in practice, the $[\mathrm{DO}]$ may be non-uniform, being almost saturated in some locations where there is a high local $k_{L} a$, and having a low [DO] in regions with poor gas dispersion. It may be concluded that simple volume averages of $k_{L} a$ from CFD simulations, without knowledge of their correlation with local driving forces, are of little practical use; they would tend to be larger than the $\left\langle k_{L} a\right\rangle$ values obtained by experiment, or from eq.(25). However, a CFD calculation which solves the oxygen transport equation, coupled with local values of $k_{L} a$ takes this effect into account, and can serve as a more correct framework for the design and scaleup of aerated stirred tanks than methods that use eq.(25) with volume averaged quantities.

Generally, the $\left\langle k_{L} a\right\rangle$ for air-water stirred tanks is given in the following form:

$\left\langle k_{L} a\right\rangle=C_{k_{L} a}\left(P_{g} / V\right)^{a} v_{g}^{b}$

For air-water system van't Riet (1979) suggested a value of $C_{k_{I} a}=0.026, a=0.4$ and $b=0.5$ obtained from a fit to experimental measurements. These constants have been the subject of many studies and their values vary from author to author depending on the tank size and gas loading. The correlations in eq.(26) are reported to be able to predict satisfactorily the $\left\langle k_{L} a\right\rangle$ of similar size vessels, but they do not necessarily apply for scale-up to an industrially sized tank (Lines, 2000; Stenberg and Andersson, 1988). 
Comparison between the $\left\langle k_{L} a\right\rangle$ estimated using the eq.(26) and the model evaluated in this work (using the [DO] evolution at the impeller region) is presented in Table 3. Higbie's model was found to give a closer prediction of $\left\langle k_{L} a\right\rangle$ compared to the value estimated using eq.(26), while Danckwerts's model consistently gave a slightly smaller $\left\langle k_{L} a\right\rangle$ value except for case 5 where the mean bubble size is larger than $5 \mathrm{~mm}$. The relative error from the $\left\langle k_{L} a\right\rangle$ value obtained from eq.(26) and the CFD simulations ranged from 3\% to 35\%, with a larger error shown for the bigger vessel. The correlations in eq.(26) are known to be problematic when applied to tanks of different size from that of the original experiments. For instance, Garcia-Cortes et al. (2004) reported a deviation up to $18 \%$ from their experimental measurement; earlier Zhu et al. (2001) reported about 20\% discrepancy. The differences in the CFD simulations might also be attributed to the poor prediction of $\varepsilon$ by the $k-\varepsilon$ turbulence model employed in this study, especially in the highly anisotropic region around the impeller. The dissipation rate can affect the mass transfer prediction in two ways: firstly, it affects the bubble interfacial area because $\varepsilon$ is used in the breakage and coalescence kernel and secondly, $k_{L}$ is directly affected when the surface renewal model is applied.

The $\left\langle k_{L} a\right\rangle$ obtained from eq. (26) is also consistently shown to be somewhat smaller than the volume averaged $k_{L} a$ (see Table 3 ). These two quantities are in fact a different measure of the mass transfer coefficient, since as noted above $\left\langle k_{L} a\right\rangle$ takes into account the effect of the driving force on the overall mass transfer rate, whereas the volume averaged value does not.

The PBM and mass transfer calculations are reasonably successful, despite the inherent difficulties of underprediction of the dissipation rate by $k-\varepsilon$ turbulence models. It should be noted however, that the kinetics of breakage and coalescence (and the mass transfer coefficient) depend on $\varepsilon^{a}$, where the exponent $|a|$ is small ( 0.25 or 0.33$)$. So a, say, $30 \%$ error in $\varepsilon$ gives rise to only about a $10 \%$ error in the kinetic rates. Single phase studies on the same grid using realizable $k-\varepsilon$ (Gimbun, 2009), show that $k$ values near the impeller blades were fairly well predicted, even though the volume integrated $\varepsilon$ was underestimated by $30 \%$. Application of a uniform scaling factor for local values of dissipation, e.g. as used by Lane et al. (2005), may then lead to an overestimate of $\varepsilon$ in regions of high breakage rate and hence was not considered approproiate in the current work.

The $\left\langle k_{L} a\right\rangle$ for an advanced gas dispersion impeller like the CD-6 appear to be slightly lower than the RDT operated at a similar $P_{g} / V, V V M$ or $v_{g}$ (in the same size of tank - 
compare cases 2 and 7 in Table 3). This finding is in agreement with the experimental work reported earlier by Zhu et al. (2001) who concluded the RDT appears to give a slightly higher $\left\langle k_{L} a\right\rangle$ than the CD-6 at the same power input. This lower $\left\langle k_{L} a\right\rangle$ obtained with the CD-6 may be attributed to several factors. The gassed power drop by CD-6 impeller is much lower than the RDT, which means it requires a lower impeller speed to achieve a similar $P_{g} / V$. The CD-6 impeller also has a slightly higher (2.3\%) gas hold-up compare to RDT (1.7\%) and this promotes slightly more bubble coalescence resulting in a smaller interfacial area and consequently lower $\left\langle k_{L} a\right\rangle$. However, the CD-6 impeller is less prone to flooding compared to the RDT.

The effect of scale-up on the mass transfer rate in gas liquid stirred tanks was also evaluated. It is impossible to keep all quantities constant at different scales, but it is feasible to maintain a couple of variables i.e. a combination of $P_{g} / V$ and either $F l_{g}, V V M$ or $v_{g}$. It is generally accepted that constant $P_{g} / V$ should be maintained, since it directly affects the local energy dissipation rate, which is the key hydrodynamic variable in the breakage and coalescence kernels. Three combinations of scale-up approaches were applied going from the $14 \mathrm{~L}$ to the 200L vessels, namely constant $P_{g} / V$ and constant $F l_{g}, V V M$ or $v_{g}$. Table 3 shows that for all 3 cases, approximately the same values of the global $\left\langle k_{L} a\right\rangle$ were obtained from the CFD-PBM caluclation. If the Higbie's model is employed for the evaluation purpose, a similar $\left\langle k_{L} a\right\rangle$ level is more likely to achieved by keeping the $P_{g} / V$ and $V V M$ constant, i.e. this rule provides a more conservative design. This might explain why in many cases of bioreactor scale-up, constant $V V M$ yields a more favourable result. None of the scale-up approaches evaluated in this work could maintain the $\left\langle k_{L} a\right\rangle$ perfectly at the same level if the Danckwerts model was employed: maintaining constant $v_{g}$ gave a slight reduction in $\left\langle k_{L} a\right\rangle$, whereas constant $V V M$ led to a slight increase. The approach outlined by eq.(26) which is based on keeping the $P_{g} / V$ and $v_{g}$ constant (case 6) does not necessarily yield a similar $\left\langle k_{L} a\right\rangle$ for a larger tank; the CFD predictions shown here give around a 10-20\% lower $\left\langle k_{L} a\right\rangle$ value for Higbie and Danckwerts models, but this is within the likely experimental error of the empirical correlations. 


\section{CONCLUSION}

A comprehensive method via CFD-PBM for modelling aerated stirred tanks has been developed. The CFD-PBM method with a drag model suitable for spherical and distorted bubbles is shown to be a better approach for modelling the gas-liquid flows in stirred tanks, than simply assuming a uniform bubble size. The power number, local bubble sizes, dissolved oxygen concentration and the mean velocities of the two-phase flow have been predicted satisfactorily in correspondence with experimental data taken from the literature. There is no significant difference between the $\left\langle k_{L} a\right\rangle$ estimated using the [DO] evolution at the impeller region, compared to those obtained at other spatial positions, for the sizes of tank studied in this work (up to 200L). The $\left\langle k_{L} a\right\rangle$ predicted using correlation, such as eq.(26), which suggest a dependence on $P_{g} / V$ and $v_{g}$ must be used with care because they may not be applicable for vessels of a different size to those from which the original correlation was derived. The scaleup of gas-liquid stirred tanks remains a very challenging task. For the small scale up factor used here (linear scaling by $\times 2.4$, or volume scaling by $\times 14$ ), all three rules gave approximately similar $\left\langle k_{L} a\right\rangle$ values. The most conservative approach was to keep both the $P_{g} / V$ and $V V M$ constant, which in the CFD-PBM computations discussed here led to a slightly larger value of $\left\langle k_{L} a\right\rangle$ at larger scale; in contrast, constant $P_{g} / V$ and $v_{g}$ led to a slight reduction in the rate of mass transfer at larger scale.

\section{ACKNOWLEDGEMENT}

JG acknowledges a scholarship from Ministry of Higher Education, Malaysia, and Universiti Malaysia Pahang. We also wish to thanks Dr. D. L. Marchisio for sharing the single phase QMOM UDF which led to the implementation of the multiphase QMOM UDF in this work.

\section{Notation}

$\begin{array}{ll}a & \text { interfacial area per unit volume } \\ a\left(L_{i}\right) & \text { breakage kernel } \\ b\left(k, L_{i}\right) & \text { daughter bubble distribution function } \\ C_{o}^{*} & \text { oxygen solubility in water } \\ C_{D} & \text { drag coefficient } \\ C_{o} & \text { oxygen concentration in water } \\ D & \text { impeller diameter } \\ {[\mathrm{DO}]} & \text { dissolve oxygen concentration } \\ d_{32} & \text { sauter mean diameter }\end{array}$




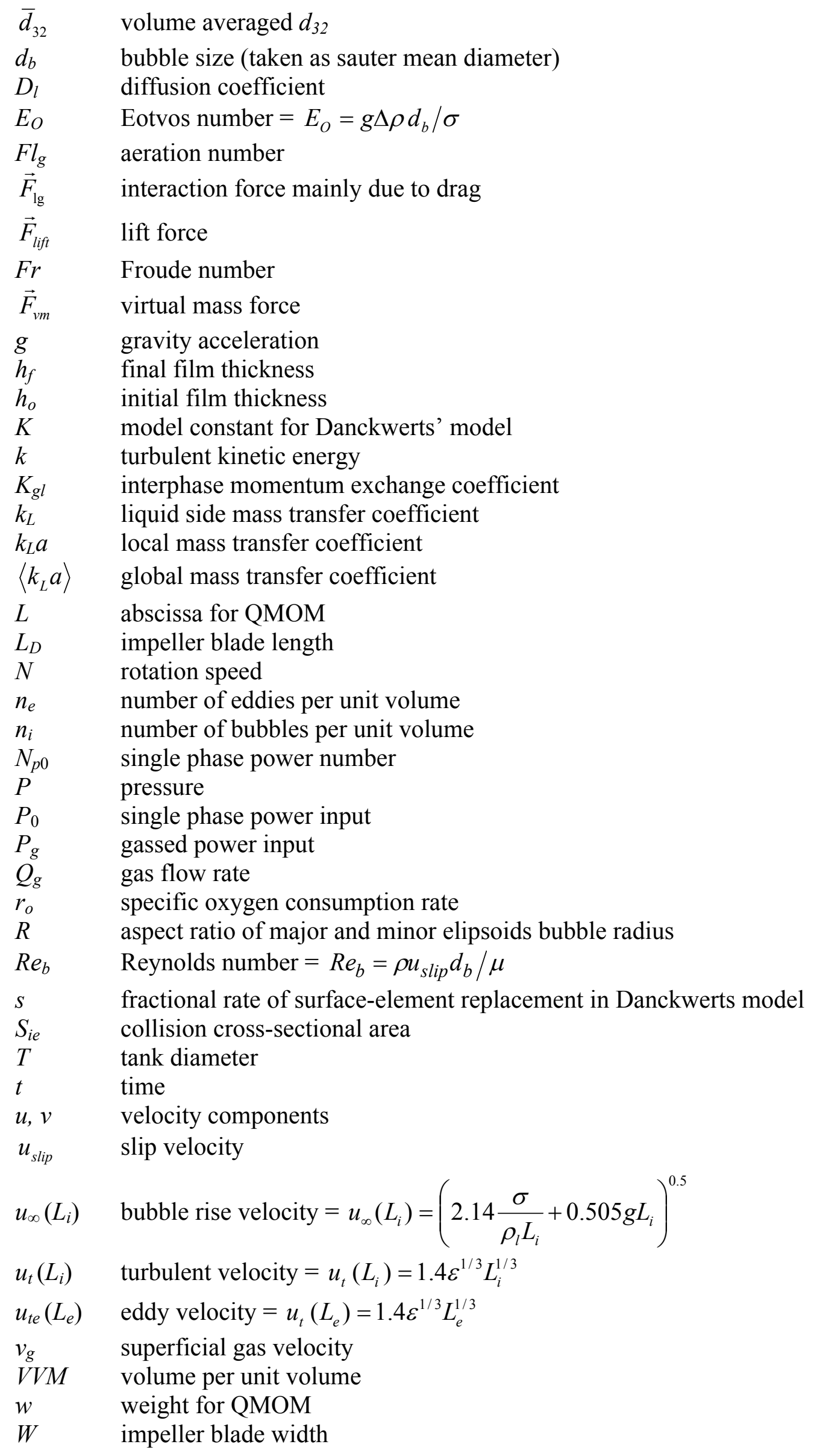




$\begin{array}{ll}\text { Greek } & \\ v_{l} & \text { kinematic viscosity } \\ \theta_{i e} & \text { collision rate of bubbles with turbulent eddies } \\ \kappa_{i} & \text { break-up efficiency } \\ \lambda\left(L_{i}, L_{j}\right) & \text { bubble collision eficiency } \\ \omega\left(L_{i}, L_{j}\right) & \text { bubble collision frequency } \\ \mu_{k} & \text { moments of the bubble size distribution } \\ \beta\left(L_{i}, L_{j}\right) & \text { coalescence kernel } \\ \varepsilon & \text { turbulent dissipation rate } \\ \Gamma & \text { torque } \\ \Delta t & \text { impeller thickness } \\ \text { Subscripts } \\ b & \text { bubble } \\ \text { dense } & \text { dense bubble } \\ g & \text { gas } \\ l & \text { liquid }\end{array}$

\section{REFERENCES}

1. Alves, S.S., Maia, C.I., Vasconcelos, J.M.T. and Serralheiro, A.J., 2002, Bubble size in aerated stirred tanks, Chem. Eng. J., 89: 109-117.

2. Andersson, R., and Andersson, B., 2006, On the breakup of fluid particles in turbulent flows, AIChE J., 52: 2020-2030.

3. Bakker, A. and Van den Akker, H.E.A., 1994, A computational model for the gas-liquid flow in stirred reactors, Chem. Eng. Res. Des. 72: 594-606.

4. Barigou, M. and Greaves, M., 1992, Bubble-size distributions in a mechanically agitated gasliquid contactor, Chem. Eng. Sci., 47: 2009-2025.

5. Behzadi, A., Issa, R.I. and Rusche, H., 2004, Modelling of dispersed bubble and droplet flow at high phase fractions, Chem. Eng. Sci., 59: 759-770.

6. Bordel, S., Mato, R. and Villaverde, S., 2006, Modeling of the evolution with length of bubble size distributions in bubble columns, Chem. Eng. Sci., 61: 3663-3673.

7. Brucato, A., Grisafi, F. and Montante, G., (1998), Particle drag coefficient in turbulent fluids, Chem. Eng. Sci., 45: 3295-3314.

8. Bujalski, W., Nienow, A.W., Chatwin, S. and Cooke, M., 1987, Dependency on scale of power numbers of rushton disc turbines, Chem. Eng. Sci., 42: 317-326.

9. Chen, P., Sanyal, J. and Dudukovic, M.P., 2005, Numerical simulation of bubble columns flows: effect of different breakup and coalescence closures, Chem. Eng. Sci., 60: 1085-1101.

10. Chesters, A.K., 1991, The modeling of coalescence processes in fluid-liquid dispersions, Trans IChemE, 69: 259-270.

11. Clift, R., Grace, J.R. and Weber, M.E., 1978, Bubbles, drops and particles, Academic Press, New York.

12. Danckwerts, P.V., 1951, Significance of liquid-film coefficients in gas absorption, Ind. Eng. Chem. 43: 1460-1467.

13. Deen, N.G., Solberg, T. and Hjertager, B.H., 2002, Flow generated by an aerated Rushton impeller: two-phase PIV experiments and numerical simulations, Can. J. Chem. Eng., 80: 638652. 
14. Derksen, J.J., Doelman, M.S. and Van den Akker, H.E.A., 1999, Three-dimensional LDA measurements in the impeller region of a turbulently stirred tank, Exp. Fluids, 27: 522-532.

15. Ducoste, J.J., Clark, M.M., 1999, Turbulence in flocculators: Comparison of measurements and CFD simulations, AIChE J., 45: 432-436.

16. FLUENT, 2006, FLUENT 6.3 User's Guide.

17. Gezork, K.M., Bujalski, W., Cooke, M. and Nienow, A.W., 2000, The transition from homogeneous to heterogeneous flow in a gassed stirred vessel, Chem. Eng. Res. Des., 78A: 363370 .

18. Gimbun, J. (2009), Scale-up of stirred bioreactors using coupled computational fluid dynamics and population balance modelling, $\mathrm{PhD}$ thesis, Department of Chemical Engineering, Loughborough University.

19. Gordon, R.G., 1968, Error bounds in equilibrium statistical mechanics, J. Math. Phys., 9: 655663.

20. Grenville, R.K. and Nienow, A.W., (2004), Blending of miscible liquids, in Paul, E.L., Atiemo-Obeng, V. and Kresta, S.M. (eds). The Handbook of Industrial Mixing (Wiley, New York, USA).

21. Guet, S., Luther, S. and Ooms, G., 2005, Bubble shape and orientation determination with a fourpoint optical fiber probe, Exp. Therm. Fluid. Sci., 29: 803-812.

22. Higbie, R., 1935, The rate of absorption of a pure gas into a still liquid during short periods of exposure, Trans. Am. Inst. Chem. Engrs., 31: 364-389.

23. Ishii, M., and Zuber N., 1979, Drag coefficient and relative velocity in bubbly, droplet or particulate flows, AIChE. J., 25: 843-855.

24. Kennard, E.H., 1938, Theory of gases, McGraw-Hill, New York.

25. Kerdouss, F., Bannari, A., and Proulx, P., 2006, CFD modelling of gas dispersion and bubble size in a double turbine stirred tank, Chem. Eng. Sci., 61: 3313-3332

26. Kerdouss, F., Bannari, A., Proulx, P., Bannari, R., Skrga, M. and Labrecque, Y., 2008, Two-phase mass transfer coefficient prediction in stirred vessel with a CFD model, Comput. Chem. Eng., 32: 1943-1955.

27. Khopkar, A.R. and Ranade, V.V., 2006, CFD simulation of gas-liquid stirred vessel: VC, S33, and L33 flow regimes, AIChE J., 52: 1654-1672.

28. Kolmogorov, A.N., 1941, The local structure of turbulence in incompressible viscous fluid for very large Reynolds numbers. Doklady Akademii Nauk SSSR, 30: 301-305.

29. Laakkonen, M., Moilanen, P., Alopaeus, V., Aittamaa, J., 2007a, Modelling local bubble size distributions in agitated vessels, Chem. Eng. Sci., 62: 721-740.

30. Laakkonen, M., Moilanen, P., Miettinen, T., Saari, K., Honkanen, M., Saarenrinne, P. and Aittamaa, J., 2005, Local bubble size distributions in agitated vessel comparison of three experimental techniques, Chem. Eng. Res. Des., 83: 50-58.

31. Laakkonen, M., Moilanen, P., Alopaeus, V. and Aittamaa, J., 2007b, Modelling local gas - Liquid mass transfer in agitated vessels, Chem. Eng. Res. Des., 85: 665-675.

32. Lamont, J.C. and Scott, D.S., 1970, An eddy cell model of mass transfer into the surface of a turbulent liquid, AIChE J., 16: 513-519.

33. Lane, G.L., Schwarz, M.P. and Evans, G.M., 2002, Predicting gas-liquid flow in a mechanically stirred tank, Appl. Math. Model., 2: 223-235

34. Lane, G.L., Schwarz, M.P. and Evans, G.M., 2005, Numerical modelling of gas-liquid flow in stirred tanks, Chem. Eng. Sci., 60: 2203-2214.

35. Lehr, F., Millies, M. and Mewes, D., 2002, Bubble size distributions and flow fields in bubble columns, AIChE J., 48: 2426-2443

36. Lines, P.C., 2000, Gas-liquid mass transfer using surface-aeration in stirred vessels with dual impellers, Chem. Eng. Res. Des., 78: 342-347

37. Luo, H., 1993, Coalescence, breakup and liquid circulation in bubble column reactors. D.Sc. Thesis, Norwegian Institute of Technology

38. Luo, H. and Svendsen, H.F., 1996, Theoretical model for drop and bubble breakup in turbulent dispersions, AIChE J., 42: 1225-1233 
39. Marchisio, D.L., Vigil, R.D. and Fox, R.O., 2003, Quadrature method of moments for aggregation-breakage processes, J. Colloid. Interface. Sci., 258: 322-334.

40. Martínez-Bazán, C., Montañéz, J.L. and Lasheras, J.C., 1999, On the breakup of an air bubble injected into a fully developed turbulent flow. Part 1 breakup frequency, J. Fluid Mech., 401: 157182.

41. McGraw, R., 1997, Description of aerosol dynamics by the quadrature method of moments, Aerosol Sci. Tech., 27: 255-265.

42. Moilanen, P., Laakkonen, M., Visuri, O., Alopaeus, V. and Aittamaa, J., 2008, Modelling mass transfer in an aerated $0.2 \mathrm{~m} 3$ vessel agitated by Rushton, Phasejet and Combijet impellers, Chem. Eng. J., 142: 95-108.

43. Montante, G., Horn, D. and Paglianti, A., 2008, Gas-liquid flow and bubble size distribution in stirred tanks, Chem. Eng. Sci., 63: 2107-2118.

44. Morud, K.E., and Hjertager, B.H., 1996, LDA measurements and CFD modelling of gas-liquid flow in a stirred vessel, Chem. Eng. Sci., 51: 233-249.

45. Myers, K.J., Thomas, A.J., Bakker, A. and Reeder, M.F., 1999, Performance of a gas dispersion impeller with vertically asymmetric blades, Chem. Eng. Res. Des., 77: 728-730.

46. Petitti, M., Caramellino, M., Marchisio, D.L. and Vanni, M., 2007, Two-scale simulation of mass transfer in an agitated gas-liquid tank, ICMF 2007, Leipzig, 9-13 July 2007.

47. Podila, K, Al Taweel, A.M.,Koksal, M., Troshko, A. and Gupta, Y.P., 2007, CFD simulation of gas-liquid contacting in tubular reactors, Chem. Eng. Sci., 62: 7151-7162.

48. Prince, M.J., and Blanch, H.W., 1990, Bubble coalescence and break-up in air-sparged bubble columns, AIChE J., 36: 1485-1499.

49. Rotta, J.C., 1972, Turbulente Stromungen, B .G. Teubner, Stuttgart.

50. Scargiali, F., D'Orazio, F., Grisafi, F. and Brucato, A., 2007, Modelling and Simulation of GasLiquid Hydrodynamics in Mechanically Stirred Tanks, Chem. Eng. Res. Des., 85: 637-646.

51. Schiller, L. and Naumann, Z., 1935, A drag coefficient correlation, Z. Ver. Deutsch. Ing., 77: 318.

52. Schuetze, J. and Hengstler, J., 2006, Assessing aerated bioreactor performance using CFD, $12^{\text {th }}$ European Conference on Mixing, (Bologna, June 27-30), pp. 439-446.

53. Shimizu, K., Takada, S., Minekawa, K. and Kawase, Y., 2000, Phenomenological model for bubble column reactors: prediction of gas hold-ups and volumetric mass transfer coefficients, Chem. Eng. J., 78: 21-28.

54. Smith, J.M., 2006, Large multiphase reactors some open questions, Chem. Eng. Res. Des., 84(A4): 265-271.

55. Stenberg, O. and Andersson, B., 1988, Gas-liquid mass transfer in agitated vessels: Part II. Modeling of gas-liquid mass transfer, Chem. Eng. Sci., 43: 725-730.

56. Sun, H., Mao, Z.-S. and Yu, G., 2006, Experimental and numerical study of gas hold-up in surface aerated stirred tanks, Chem. Eng. Sci., 61: 4098-4110.

57. van't Riet, K., 1979, Review of measuring methods and results in nonviscous gas-liquid mass transfer in stirred vessels, Ind. Eng. Chem. Proc. Des. Dev., 18: 357-360.

58. Venneker, B.C.H., Derksen, J.J. and Van den Akker, H.E.A., 2002, Population balance modeling of aerated stirred vessels based on CFD, AIChE J., 48: 673-685.

59. Wang, W., Mao, Z.-S. and Yang, C., 2006, Experimental and numerical investigation on gas holdup and flooding in an aerated stirred tank with Rushton impeller, Ind. Eng. Chem. Res., 45: 1141-1151.

60. Warmoeskerken, M.M.C.G. and Smith, J.M., 1982, Description of the power curves of turbine stirred gas dispersions, Proc Fourth Europ Conf on Mixing, Noordwijkerhout, BHRA, Cranfield, pp. 237-246.

61. Wellek, R.M., Arawal, A.K. and Skelland, A.H.P., 1966, Shapes of liquid drops moving in liquid media, AIChE J, 12: 854-862.

62. Zhu, Y., Bandopadhayay, P.C. and Wu, J., 2001, Measurement of gas-liquid mass transfer in an agitated vessel-A comparison between different impellers, J. Chem. Eng. Jpn., 34: 579-584. 


\section{List of figures}

Fig. 1: Result from grid analysis at $z=0$ with respect to the impeller and $r / R=0.37$, a) gas tangential velocity, b) $k$ of the liquid phase

Fig. 2: Prediction of gas phase axial $(u)$ and radial $(v)$ velocity at normalised radial position (radial position, $r$ over tank radius, $R$ ) $r / R=0.37$. Schiller and Naumann (1935) drag model (CFD-PBM-SN), Ishii and Zuber (1979) drag model (CFD-PBM-IZ). Experimental data from Deen and Hjertager (2002).

Fig. 3: Prediction of liquid phase axial $(u)$ and radial velocity $(v)$ at $r / R=0.37$. Experimental data from Deen and Hjertager (2002).

Fig. 4: Prediction of local Sauter mean bubble diameter for case 2: RDT, 14 L tank, $N=700$ rpm, $Q_{g}=0.7$ vvm. A) Laakkonen et al. (2007a) measurement (bold), this work (bracket), B) Contour map of Sauter mean diameter

Fig. 5: Prediction of local Sauter mean bubble diameter for case 4: RDT, $200 \mathrm{~L}$ tank, $N=390$ rpm, $Q_{g}=0.7$ vvm. A) Laakkonen et al. (2007a) measurement (bold), this work (bracket), B) Contour map of Sauter mean diameter

Fig. 6: Prediction of local $k_{L} a$ with a RDT. A) 14 L tank (case 2), B) 200 L tank (case 3)

Fig. 7: Comparison of CFD-PBM-IZ prediction of the two-phase flow in the $14 \mathrm{~L}$ tank with a CD-6 impeller (case 7) and RDT (case 2): $P_{g} / V=1174.7 \mathrm{~W} / \mathrm{m}^{3}, Q_{g}=0.7 \mathrm{VVM}$, A) velocity vectors; contours of gas void fraction, $\mathrm{B}$ ) contours of gas void fraction at the impeller level

Fig. 8: Prediction of [DO] evolution for case 3, $N=390 \mathrm{rpm}$ and $Q_{g}=0.7 V V M$. Data points from Laakkonen et al. (2007b).

Fig. 9: Comparison between the [DO] evolution calculated using two different method at impeller level, a) case 2, $N=700 \mathrm{rpm}, Q_{g}=0.7 V V M$, b) case $4, N=365.8 \mathrm{rpm}, Q_{g}=0.37$ $V V M$

Fig. 10: Comparison between the $\left\langle k_{L} a\right\rangle$ estimated using the [DO] evolution obtained at different position inside the tank for case 5, $N=386 \mathrm{rpm}$ and $Q_{g}=0.7 V V M$. Higbie's method (bold font), Danckwerts's method (italic font)

Fig. 11: Comparison between the [DO] evolution for a different scale-up approach, a) Higbie's Method, b) Danckwerts's method

Fig. 12: Comparison between the [DO] evolution for a tank agitated by RDT (case 2) and CD6 (case-7) operating at similar $P_{g} / V, F_{l g}, V V M$ and $v_{g}$, a) Higbie's Method, b) Danckwerts's method 


\section{List of Tables}

Table 1: Prediction of the relative power number for Rushton Turbine (RDT)

\begin{tabular}{|c|c|c|c|c|c|c|c|c|c|c|}
\hline \multirow[t]{2}{*}{ Case } & \multirow{2}{*}{$\begin{array}{c}T \\
(\mathrm{~m})\end{array}$} & \multirow{2}{*}{$\begin{array}{c}D \\
(\mathrm{~m})\end{array}$} & \multirow[t]{2}{*}{$F_{l g}$} & \multirow[t]{2}{*}{$V V M$} & \multirow{2}{*}{$\begin{array}{c}v_{g} \\
(\mathrm{~cm} / \mathrm{s})\end{array}$} & \multirow{2}{*}{$\begin{array}{c}N \\
(\mathrm{rpm})\end{array}$} & \multirow{2}{*}{$\begin{array}{c}\bar{d}_{32} \\
(\mathrm{~mm})\end{array}$} & \multicolumn{3}{|c|}{ Relative power number } \\
\hline & & & & & & & & $\begin{array}{l}P_{g} / P_{0} \\
\text { eq.(24) }\end{array}$ & $\begin{array}{c}P_{g} / P_{0} \\
\text { CFD constant } d_{b}\end{array}$ & $\begin{array}{c}P_{g} / P_{0} \\
\text { CFD-PBM-IZ }\end{array}$ \\
\hline 1 & 0.26 & 0.086 & 0.030 & 0.70 & 0.30 & 513.0 & 2.8 & 0.47 & 0.44 & 0.46 \\
\hline 2 & 0.26 & 0.086 & 0.022 & 0.70 & 0.30 & 700.0 & 2.5 & 0.45 & 0.41 & 0.45 \\
\hline 3 & 0.63 & 0.210 & 0.038 & 0.70 & 0.74 & 390.0 & 5.3 & 0.42 & 0.38 & 0.43 \\
\hline 4 & 0.63 & 0.210 & 0.022 & 0.37 & 0.39 & 365.8 & 4.1 & 0.49 & 0.43 & 0.47 \\
\hline 5 & 0.63 & 0.210 & 0.038 & 0.70 & 0.74 & 386.4 & 5.3 & 0.42 & 0.39 & 0.43 \\
\hline 6 & 0.63 & 0.210 & 0.017 & 0.29 & 0.30 & 357.6 & 3.5 & 0.53 & 0.42 & 0.50 \\
\hline
\end{tabular}

Table 2: Prediction of the relative power number for CD-6

\begin{tabular}{cccccccccc}
\hline Case & $T$ & $D$ & $F_{l g}$ & $V V M$ & $N$ & $\bar{d}_{32}$ & \multicolumn{3}{c}{ Relative power number } \\
& $(\mathrm{m})$ & $(\mathrm{m})$ & & & $(\mathrm{rpm})$ & $(\mathrm{mm})$ & $\begin{array}{c}P_{g} / P_{0} \text { measured } \\
(\text { Myers et al., 1999) }\end{array}$ & $\begin{array}{c}P_{g} / P_{0} \\
\text { CFD constant } d_{b}\end{array}$ & $\begin{array}{c}P_{g} / P_{0} \\
\text { CFD-PBM-IZ }\end{array}$ \\
\hline 7 & 0.26 & 0.086 & 0.22 & 0.70 & 698 & 3.4 & 0.71 & 0.75 & 0.69 \\
\hline
\end{tabular}

Table 3: Prediction of mass transfer coefficient

\begin{tabular}{ccccccc}
\hline & Scale-up & \multicolumn{3}{c}{$\left\langle k_{L} a\right\rangle\left(\mathrm{s}^{-1}\right)$} & \multicolumn{3}{c}{ Volume averaged $k_{L} a\left(\mathrm{~s}^{-1}\right)$} \\
\cline { 3 - 6 } Case & parameter & van't Riet eq.(26) & Higbie & Danckwerts & Higbie & Danckwerts \\
\hline 2 & Base case & 0.024 & 0.023 & 0.018 & 0.031 & 0.026 \\
4 & $P_{g} / V$ and $F_{l g}$ & 0.028 & 0.022 & 0.021 & 0.026 & 0.025 \\
5 & $P_{g} / V$ and $V V M$ & 0.038 & 0.027 & 0.031 & 0.033 & 0.038 \\
6 & $P_{g} / V$ and $v_{g}$ & 0.024 & 0.018 & 0.015 & 0.022 & 0.019 \\
7 & $P_{g} / V$ and $V V M$ & 0.024 & 0.019 & 0.015 & 0.021 & 0.016 \\
& and CD-6 & 0.026 & & &
\end{tabular}




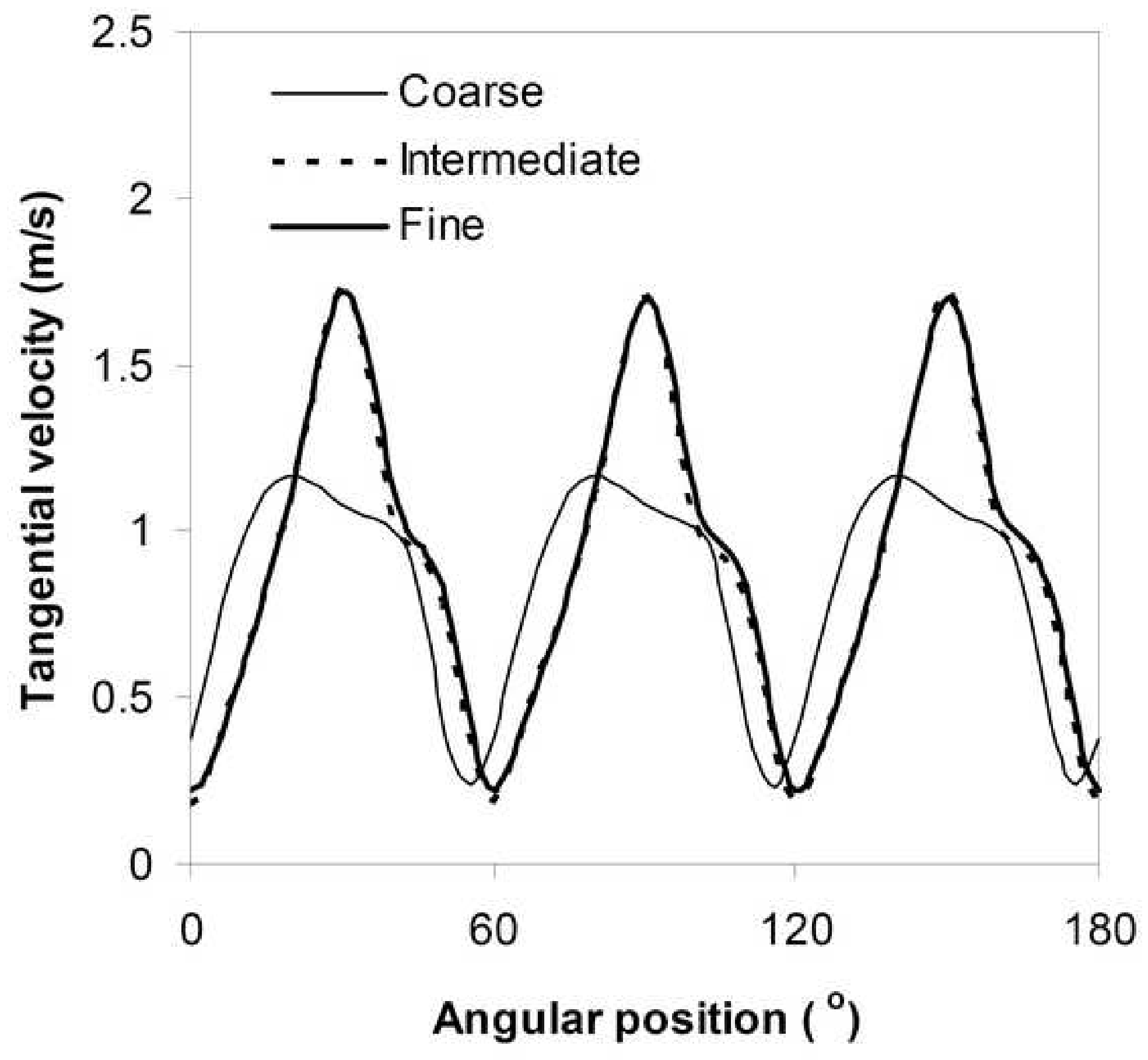




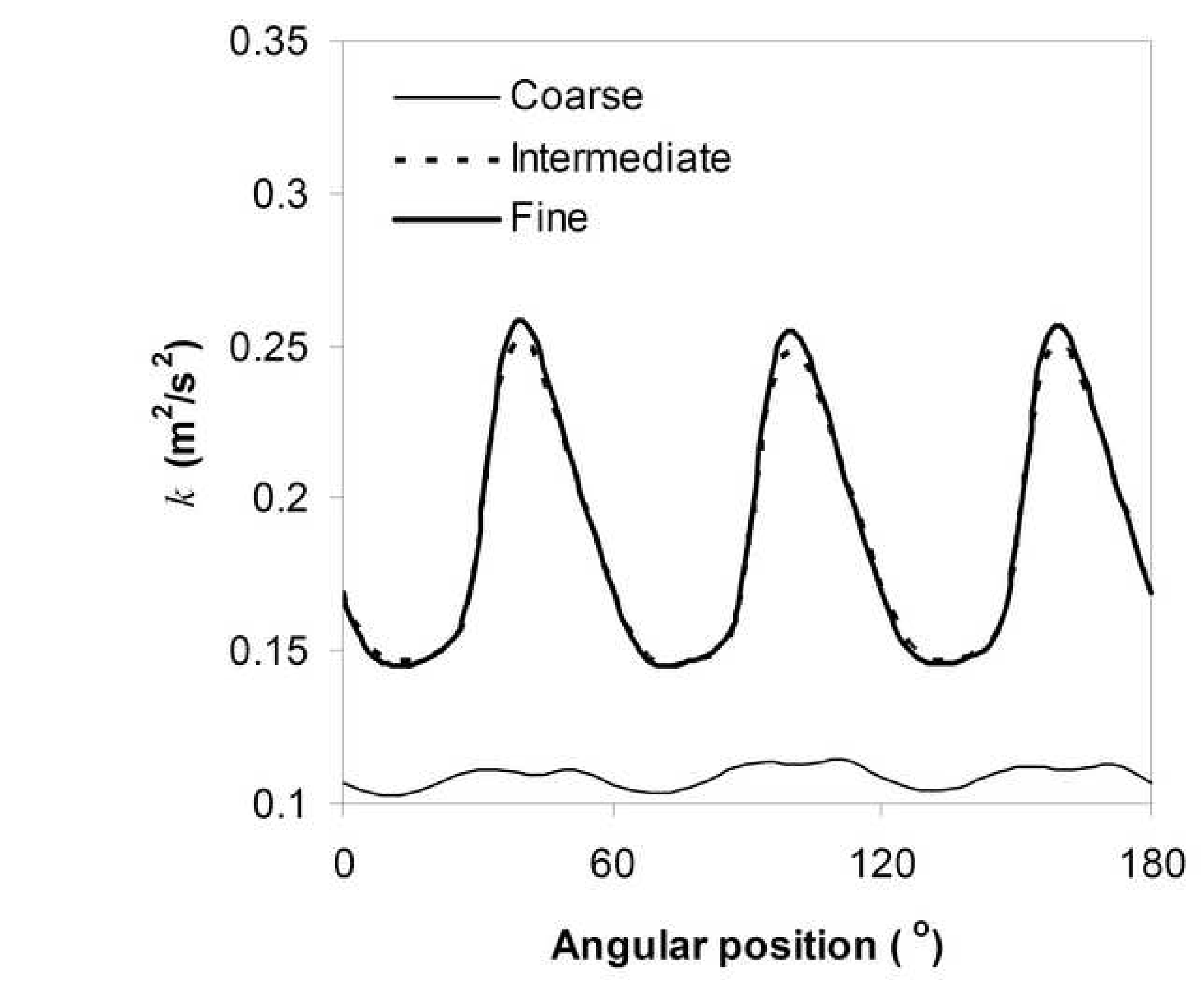



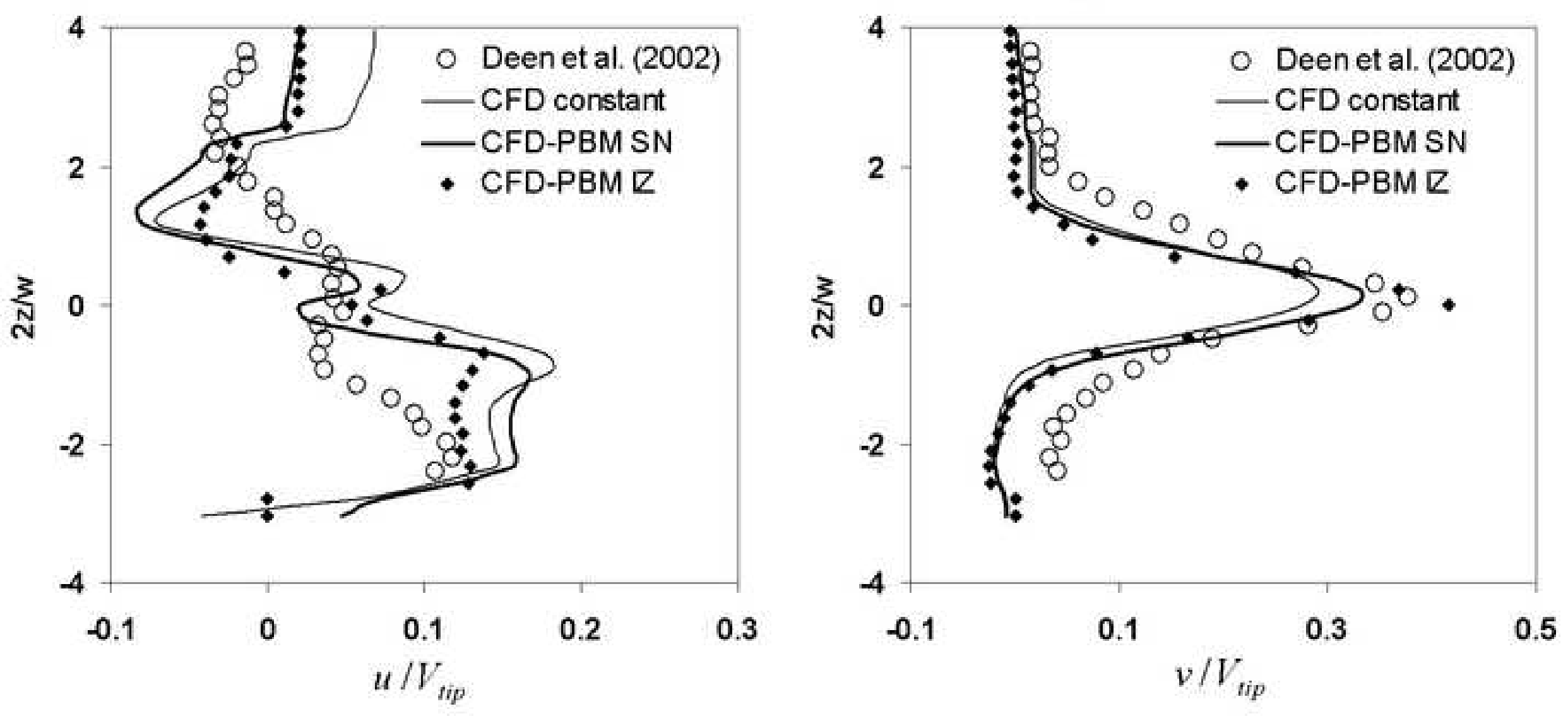

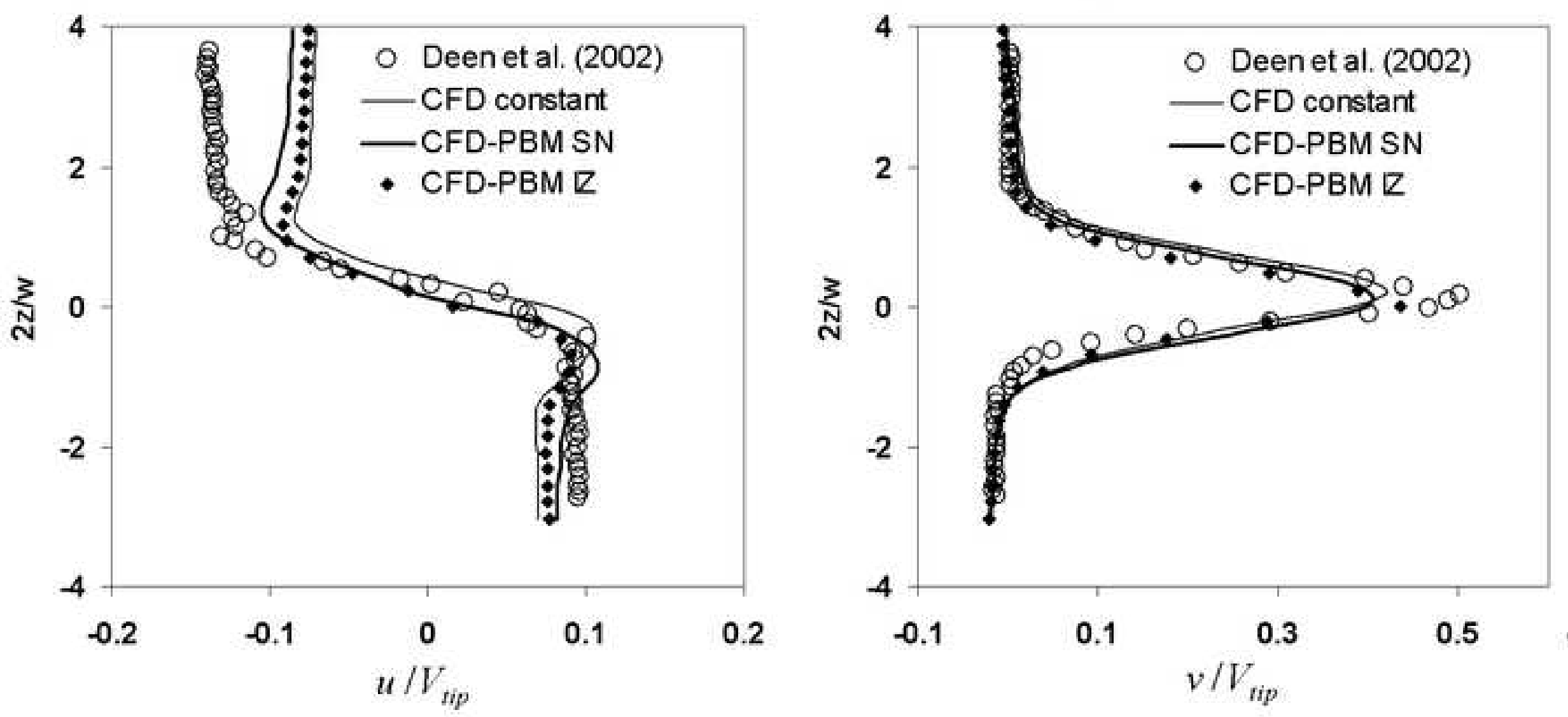


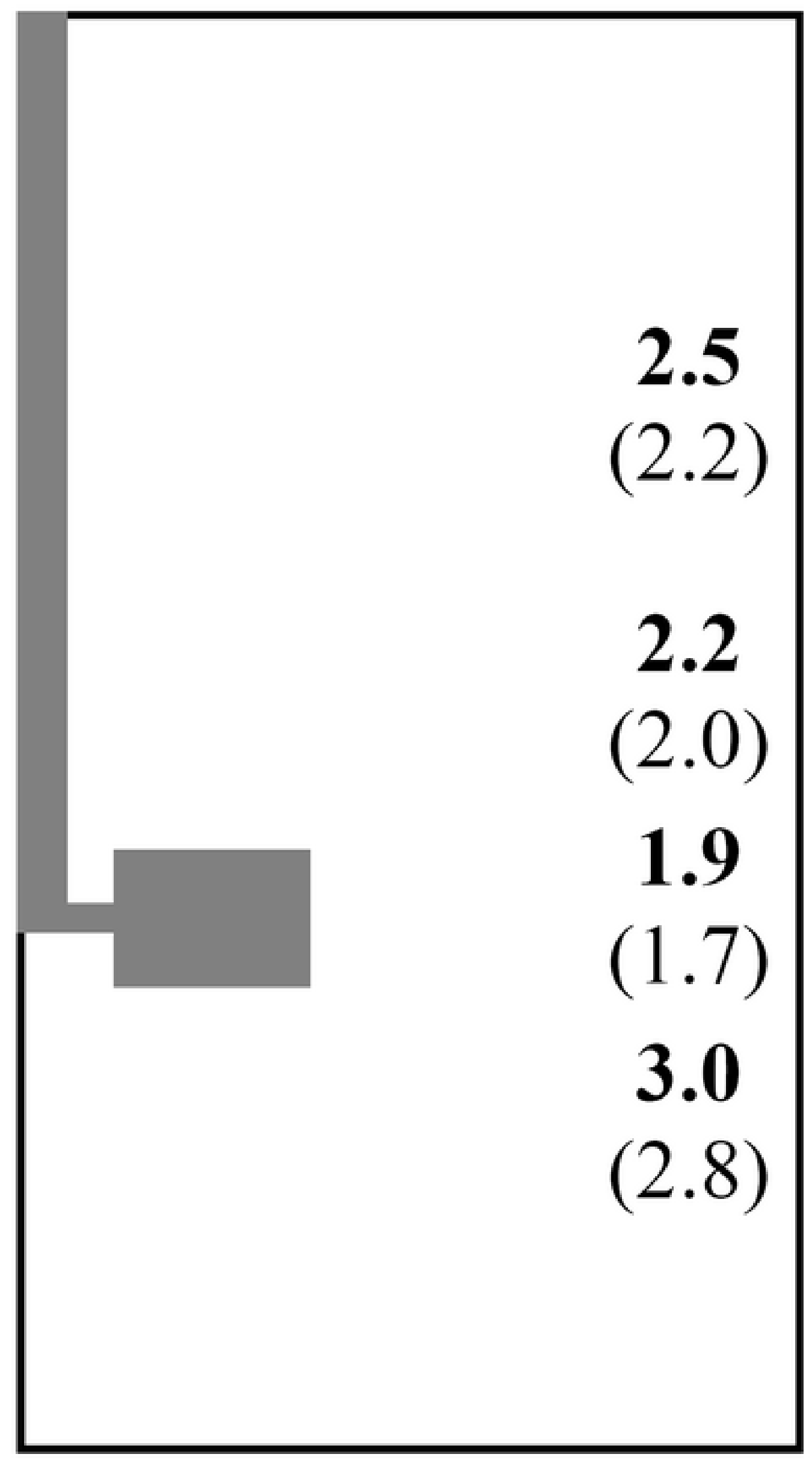

Fig 4a.TIF

2.2

(2.0)

1.9

(1.7)

3.0

(2.8)

2.5

$(2.2)$

$$
\text { . }
$$

ga.TIF 

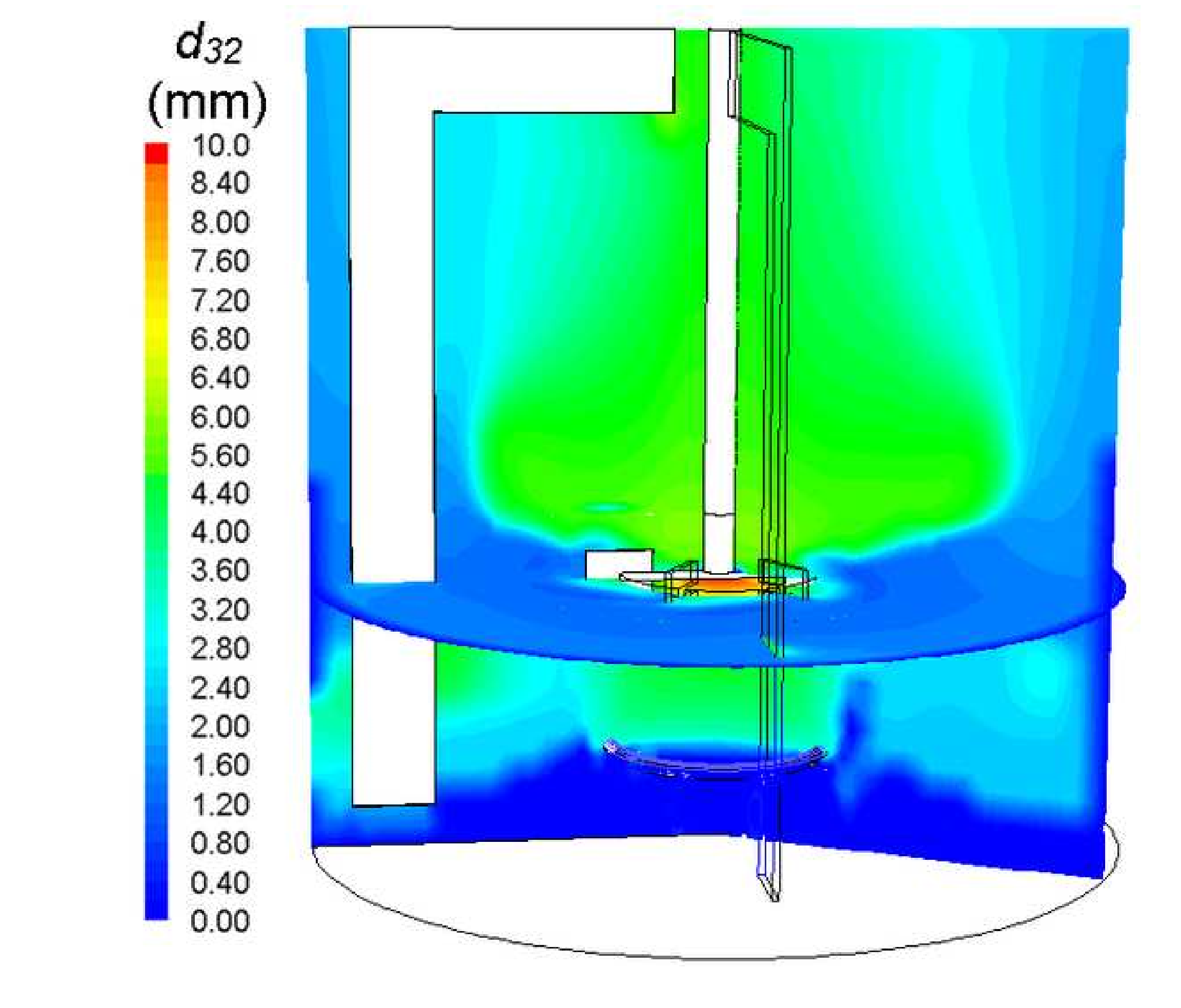


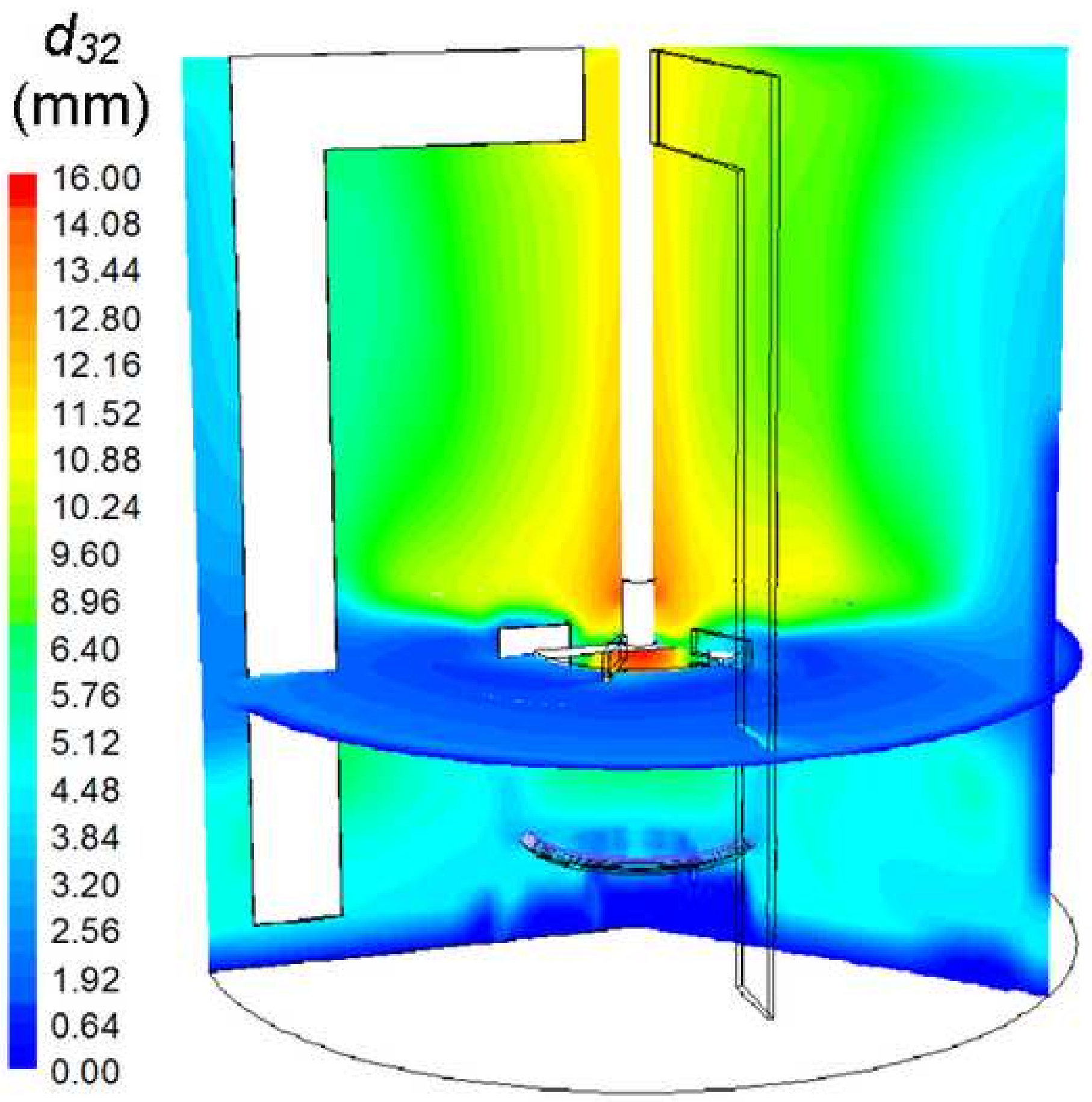




\section{$k_{L} a(1 / s)$ Higbie Danckwerts}

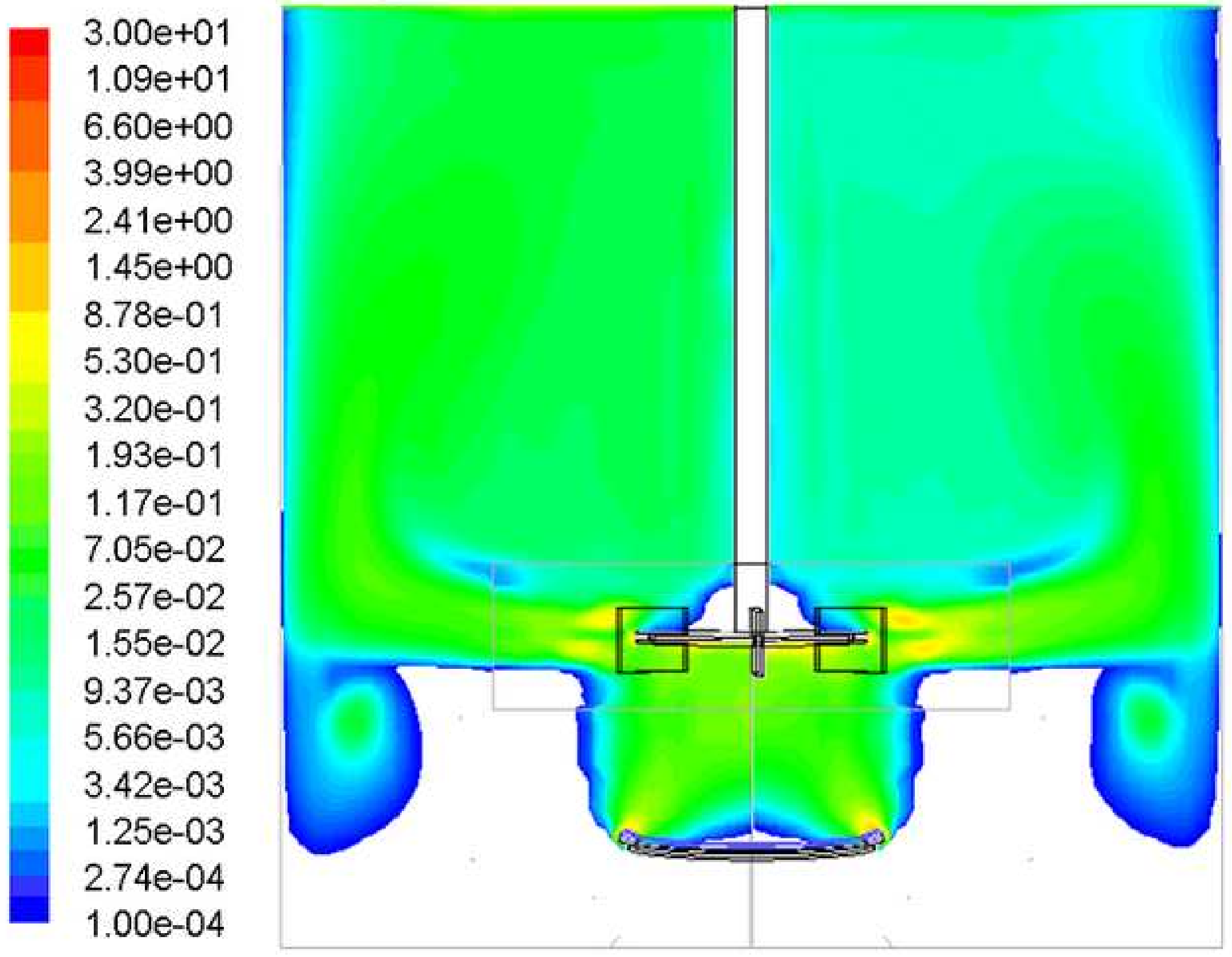


$k_{L} a(1 / s)$ Higbie

Danckwerts

$1.50 \mathrm{e}+01$
$2.23 \mathrm{e}+00$
$1.38 \mathrm{e}+00$
$5.33 \mathrm{e}-01$
$3.31 \mathrm{e}-01$
$2.05 \mathrm{e}-01$
$1.28 \mathrm{e}-01$
$7.92 \mathrm{e}-02$
$1.18 \mathrm{e}-02$
$7.30 \mathrm{e}-03$
$4.53 \mathrm{e}-03$
$2.81 \mathrm{e}-03$
$1.75 \mathrm{e}-03$
$1.08 \mathrm{e}-03$
$6.73 \mathrm{e}-04$
$4.18 \mathrm{e}-04$
$1.00 \mathrm{e}-04$

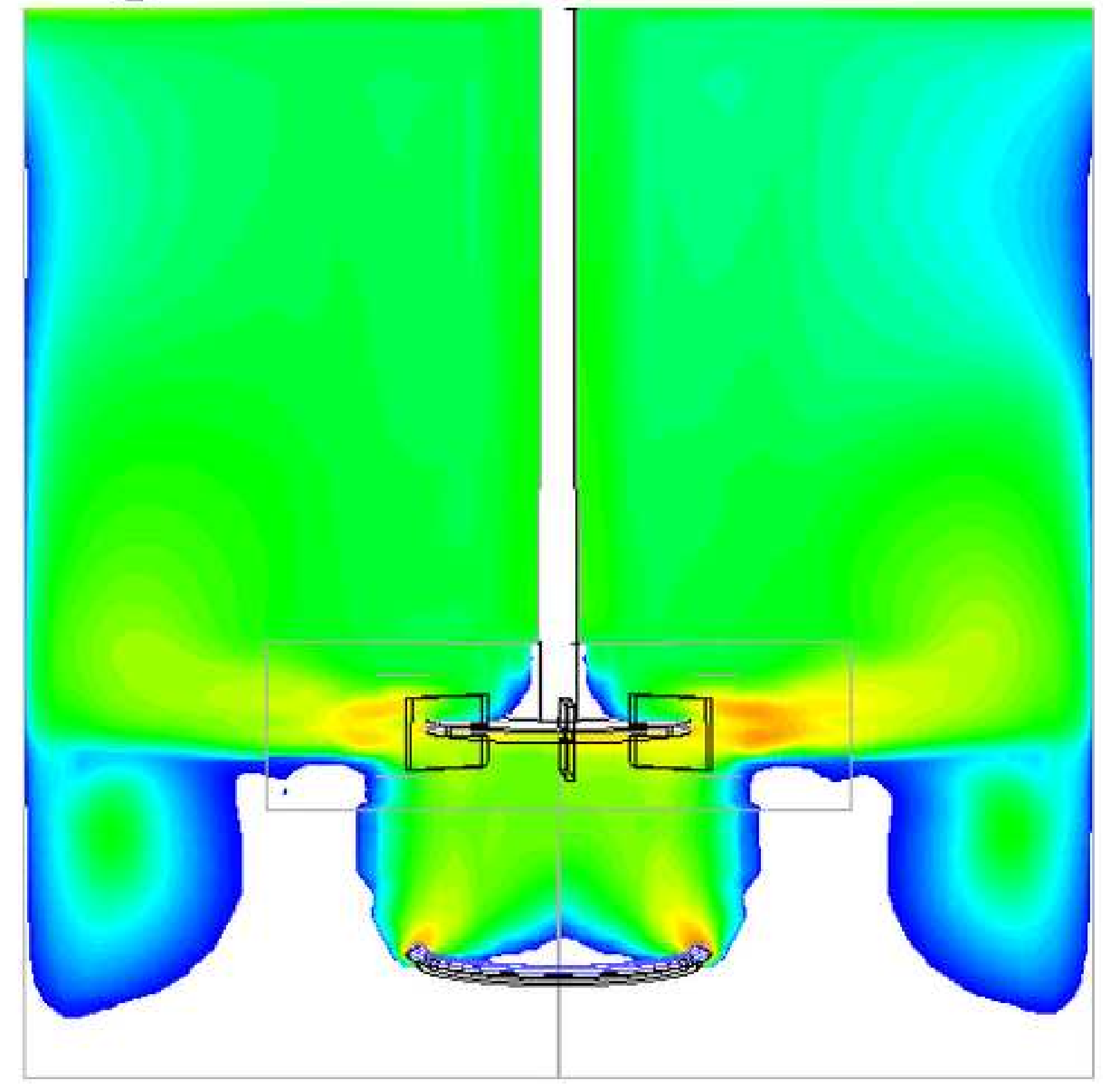


$1.00 \mathrm{e}+00$

2.34e-01

1.13e-01

$5.49 \mathrm{e}-02$

2.66e-02

$1.29 \mathrm{e}-02$

$6.23 \mathrm{e}-03$

3.02e-03

7.07e-04

$3.42 \mathrm{e}-04$

$1.88 \mathrm{e}-05$

$9.10 \mathrm{e}-06$

$4.41 \mathrm{e}-06$

2.13e-06

$1.03 \mathrm{e}-06$

$5.00 \mathrm{e}-07$

\section{CD-6}

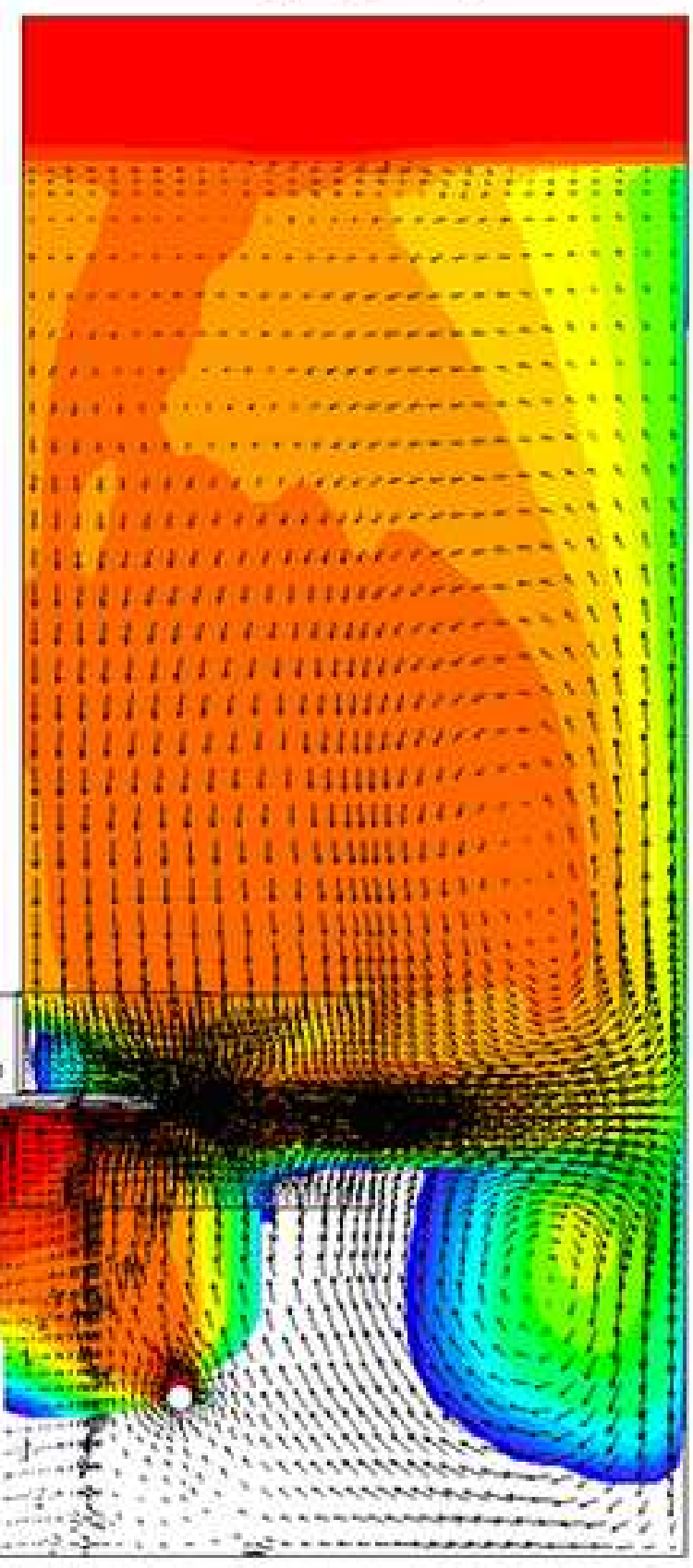

RDT

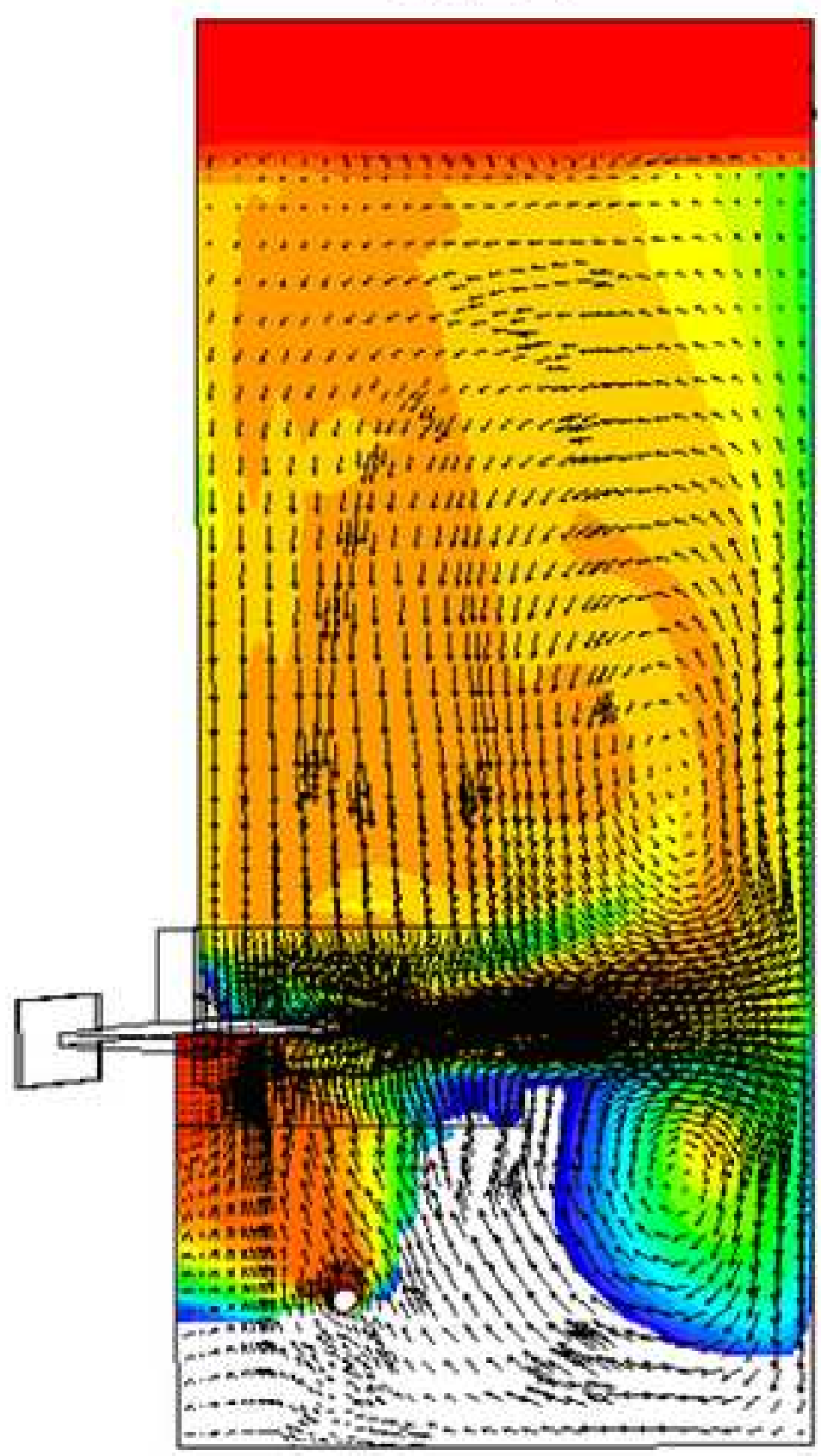




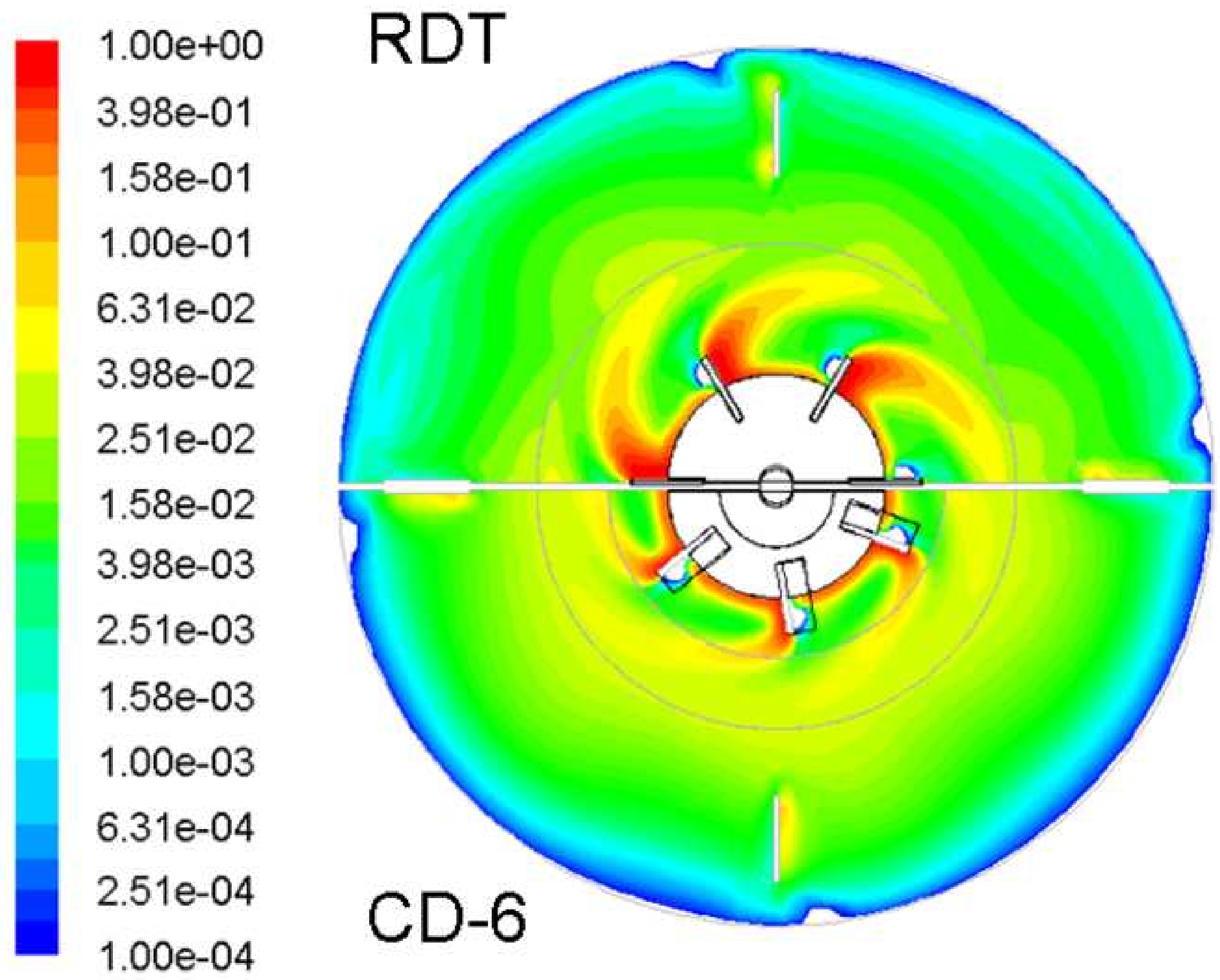




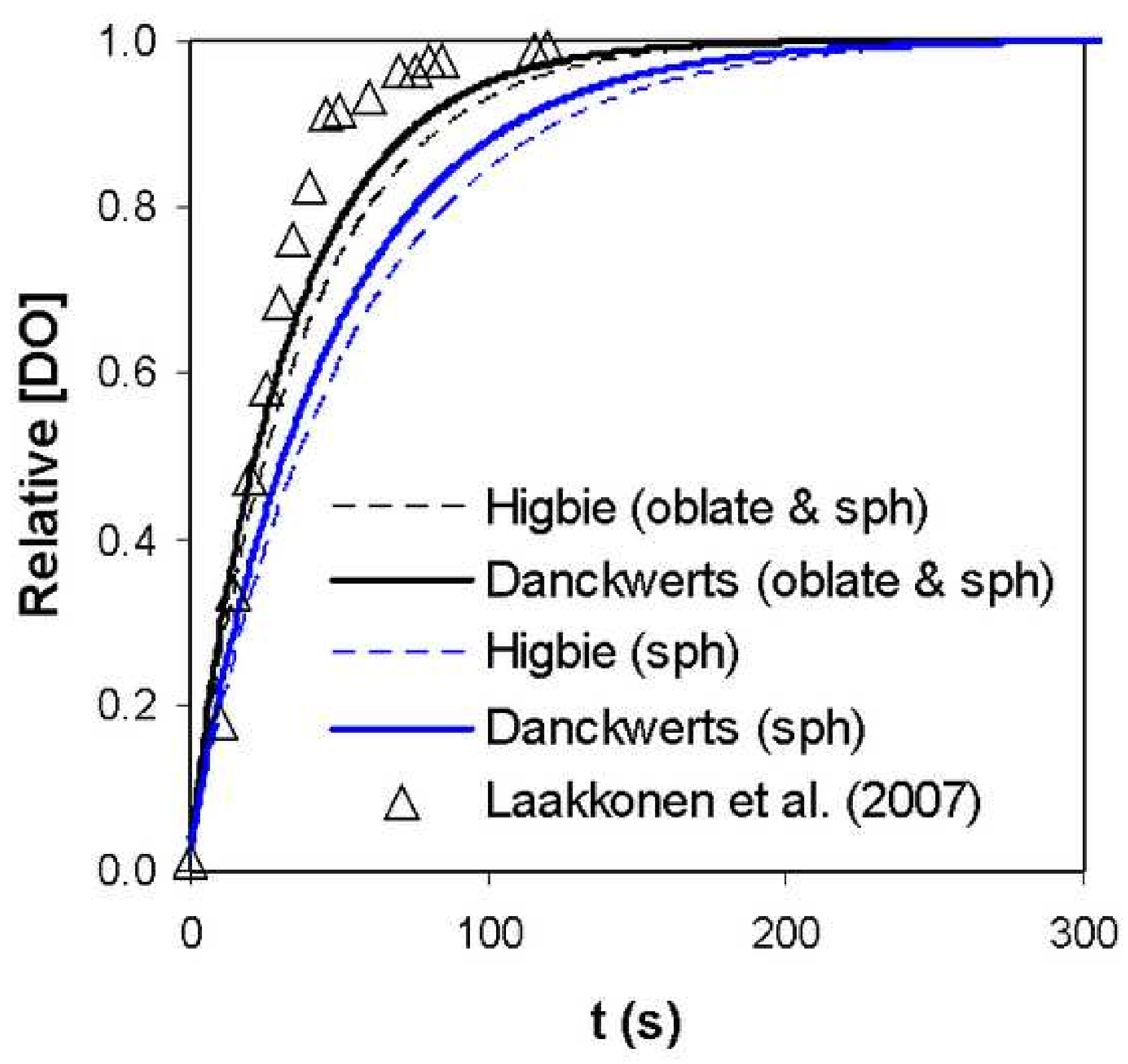




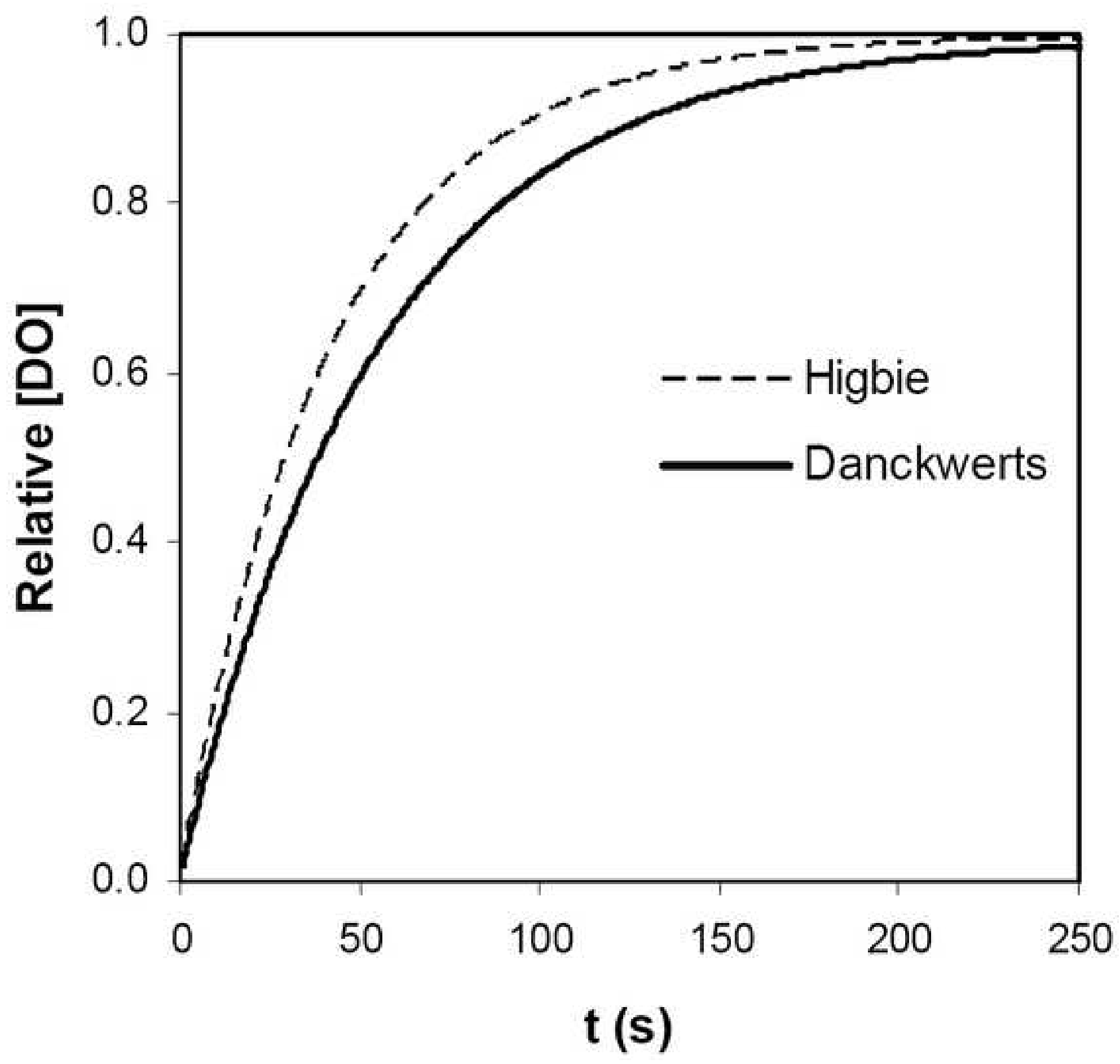




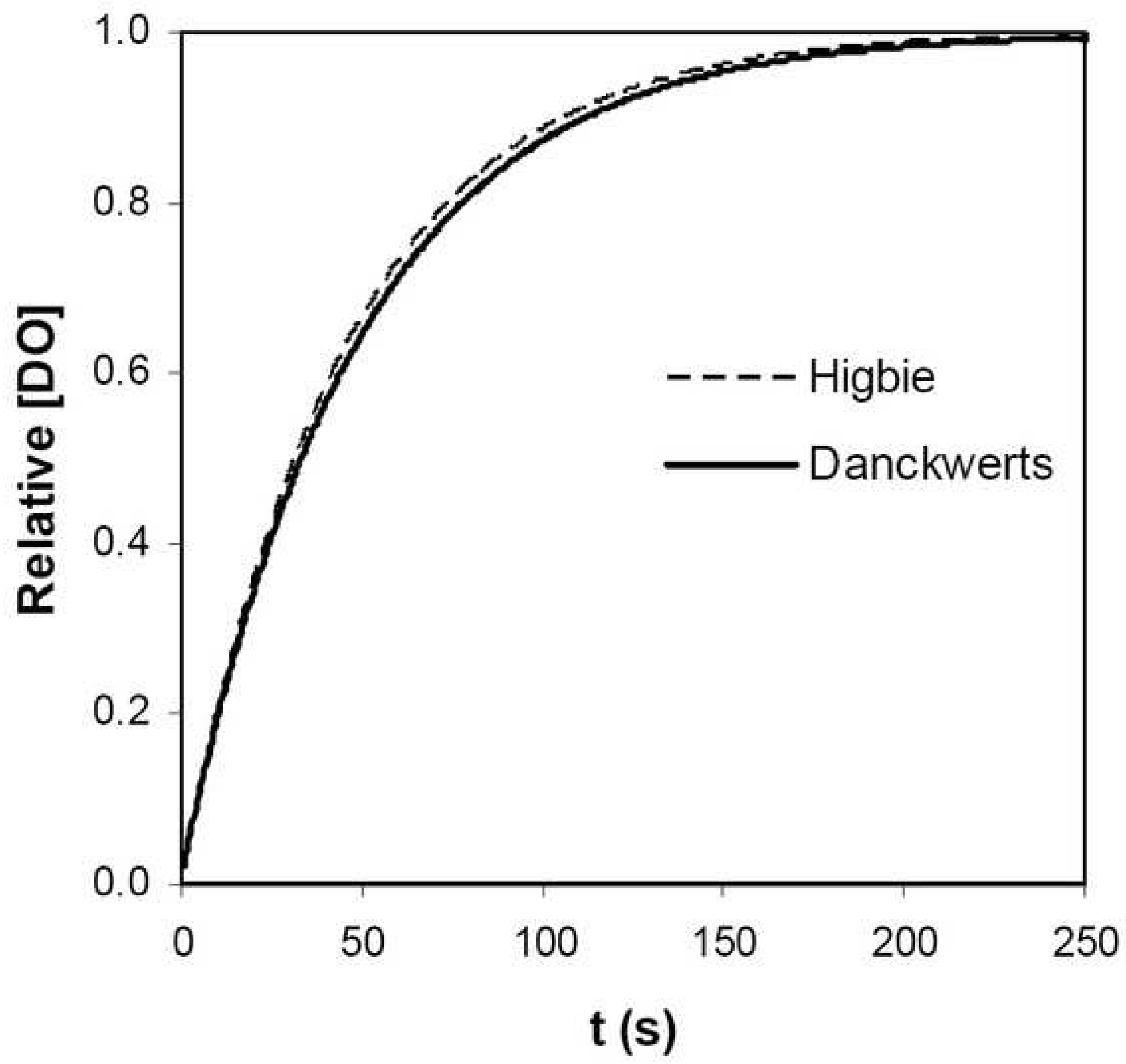




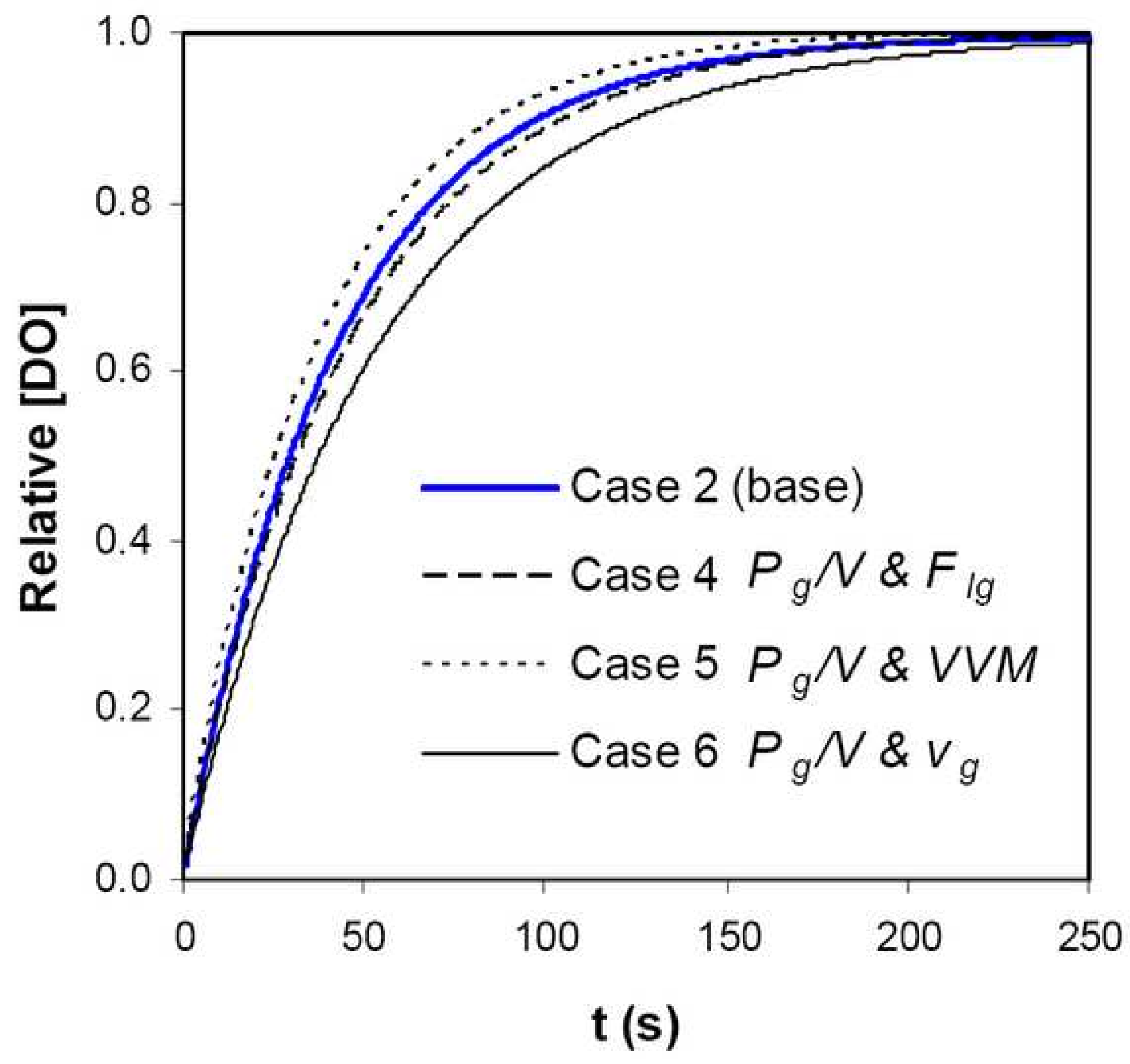




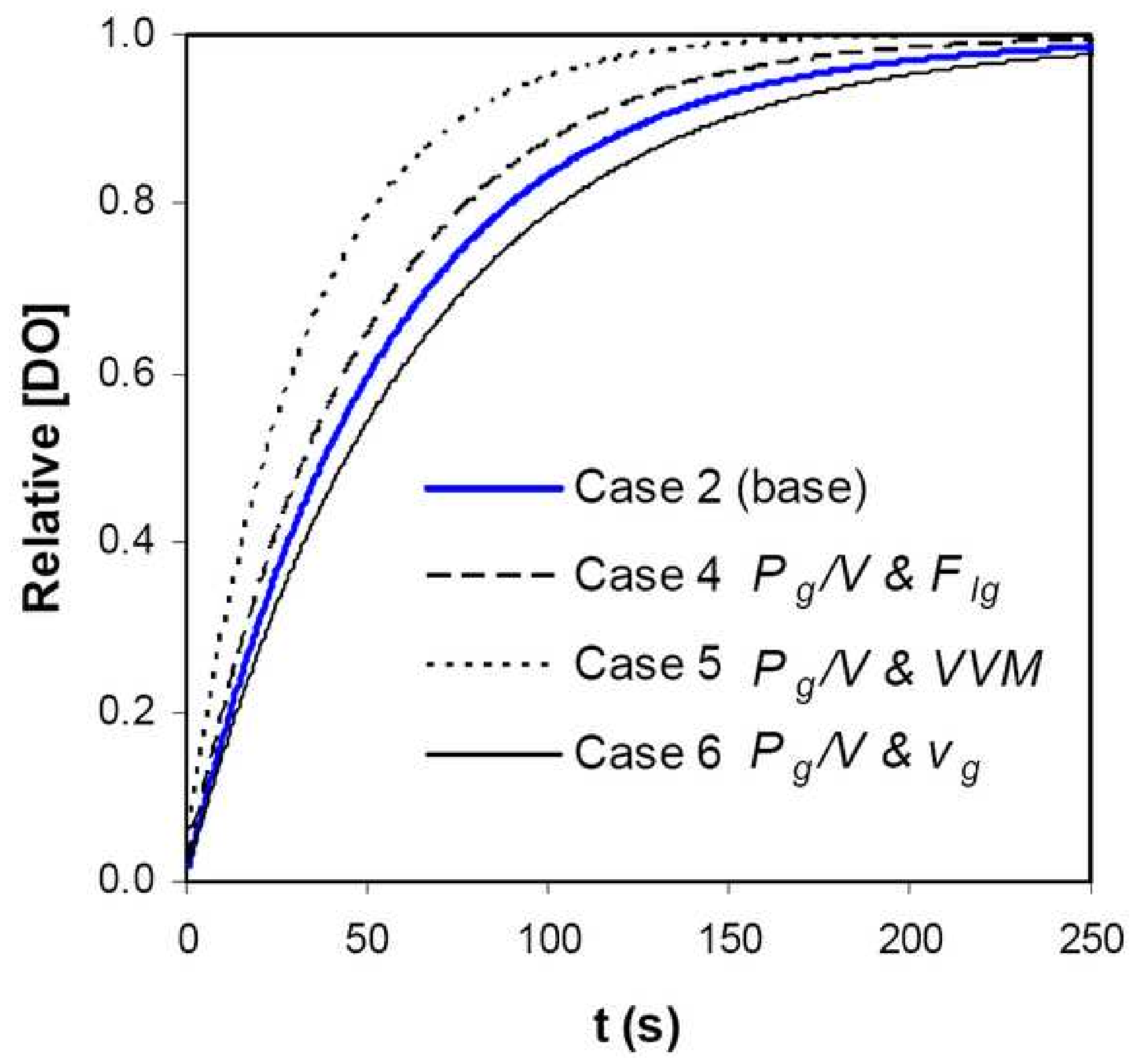




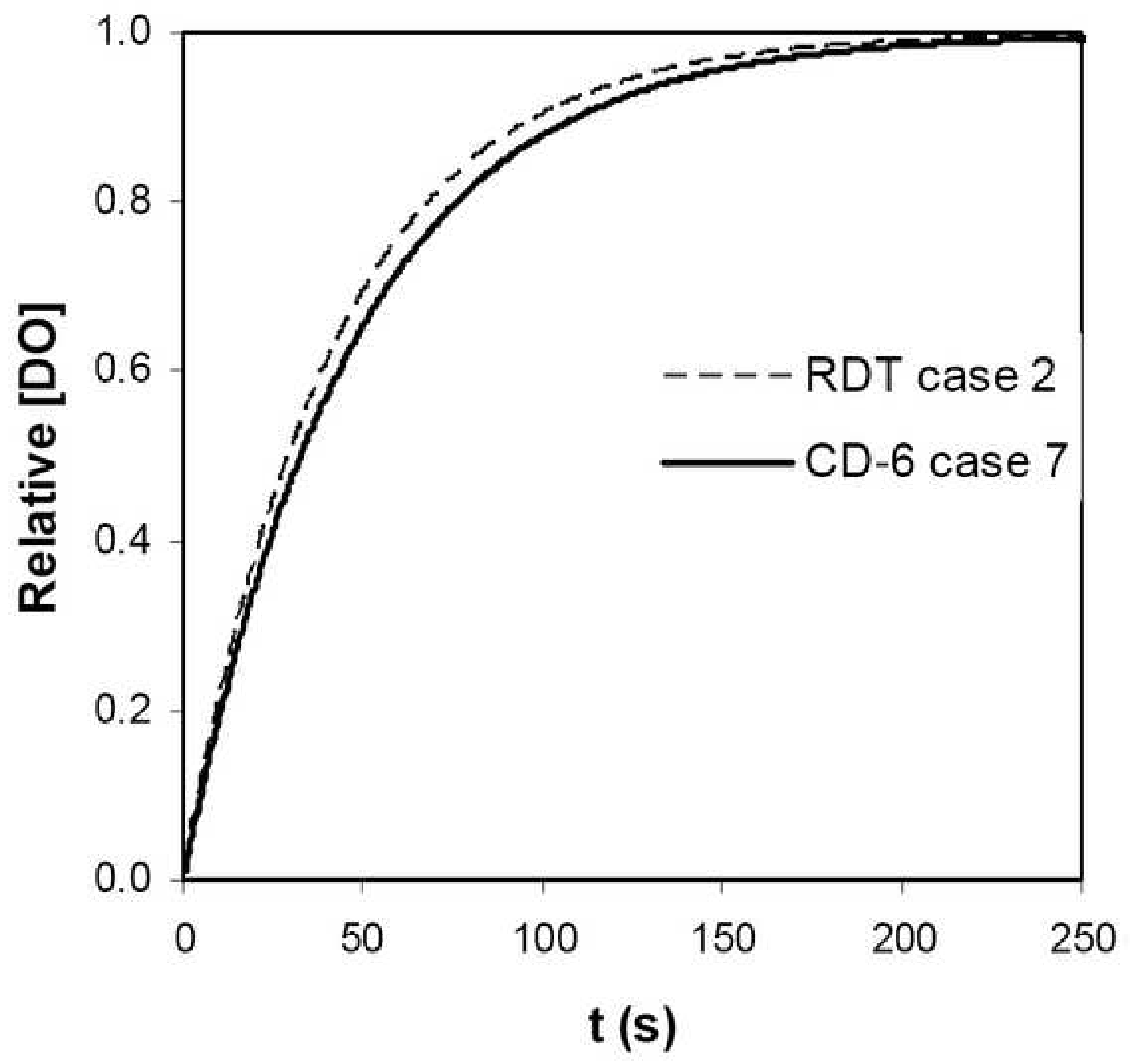




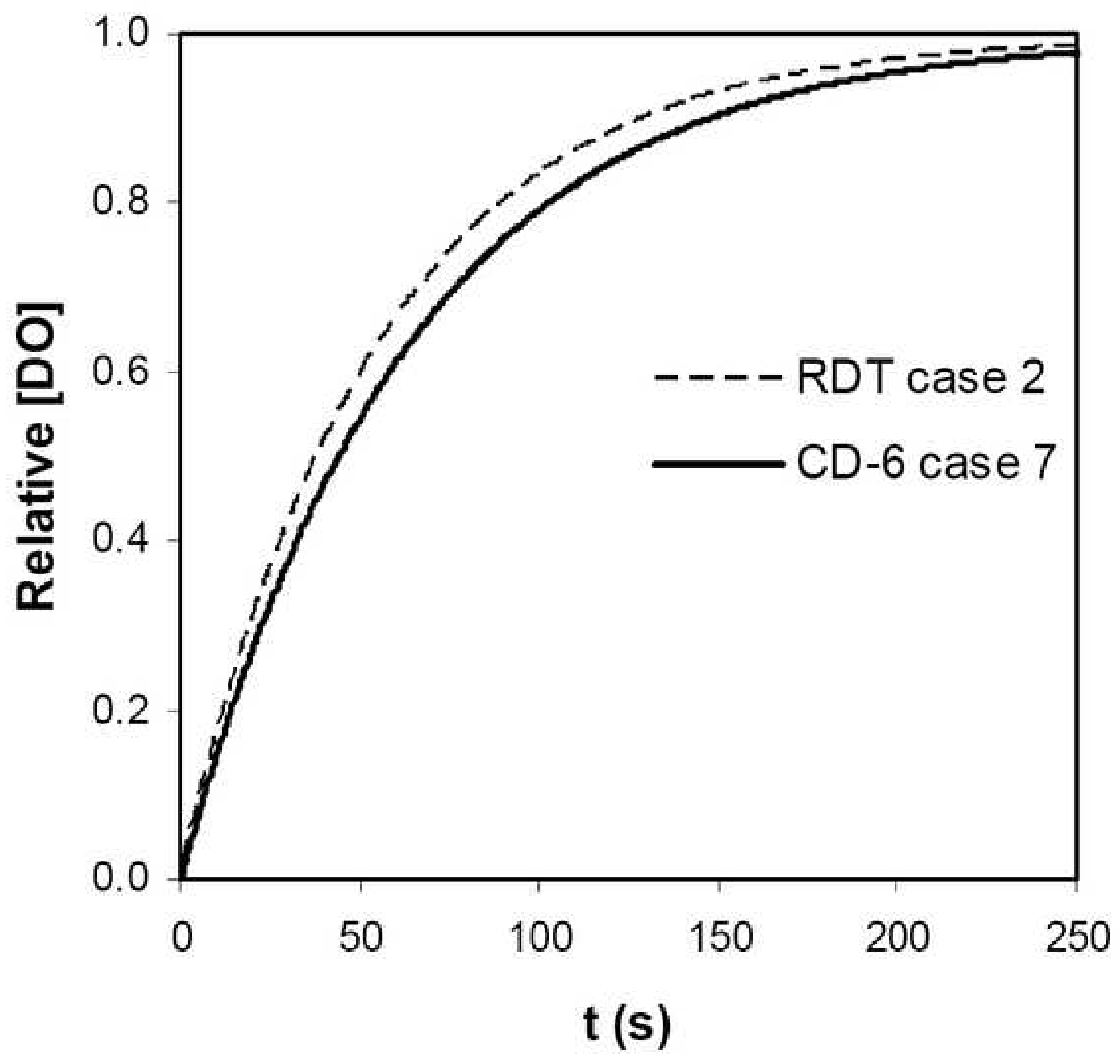

